\title{
Research on the Formation Mechanism of Surface Morphology in Three-Excitation Ultrasonic Spatial Vibration-Assisted Turning
}

\author{
Jingwei Duan \\ Northeastern University \\ Ping Zou ( $\sim$ pzou425@163.com ) \\ Northeastern University \\ Shiyu Wei \\ Northeastern University \\ Rui Fang \\ Northeastern University \\ Liting Fang \\ Northeastern University
}

\section{Research Article}

Keywords: Three-excitation ultrasonic spatial vibration-assisted turning, Response surface methodology, Surface roughness, Formation mechanism, Influencing factors

Posted Date: January 12th, 2022

DOI: https://doi.org/10.21203/rs.3.rs-1222943/v1

License: (c) (1) This work is licensed under a Creative Commons Attribution 4.0 International License. Read Full License 


\title{
Research on the formation mechanism of surface morphology in three-excitation ultrasonic spatial vibration-assisted turning
}

\author{
Jingwei Duan Ping Zou* Shiyu Wei Rui Fang Liting Fang \\ School of mechanical engineering and automation, Northeastern University, Shenyang 110819 \\ Corresponding author: Ping Zou \\ E-mail: pzou@mail.neu.edu.cn
}

\begin{abstract}
To improve the machining performance of different processing materials, a three-excitation ultrasonic spatial vibration-assisted turning system is proposed, which realizes the non-unity of the plane where the cutting trajectory of the tool is located. The influence and formation law of three-excitation ultrasonic spatial vibration-assisted turning on the surface roughness of the workpiece under different vibration parameters (amplitude) and machining parameters (cutting speed, cutting depth, and feed) were analyzed by response surface methodology. The results show that in terms of vibration parameters, the influence of ultrasonic vibration applied in the horizontal direction on surface roughness is significantly greater than that of ultrasonic vibration applied in the vertical direction, while the feed has the greatest influence on surface roughness, followed by cutting speed. The surface roughness of common turning, one-dimensional ultrasonic vibration-assisted turning, ultrasonic elliptical vibration-assisted turning, and three-excitation ultrasonic spatial vibration-assisted turning were theoretically analyzed and experimentally compared. The results show that compared with the other three turning methods, the three-excitation ultrasonic spatial vibration-assisted turning can obtain a lower surface roughness and have good machinability.
\end{abstract}

Keywords: Three-excitation ultrasonic spatial vibration-assisted turning - Response surface methodology $\cdot$ Surface roughness $\cdot$ Formation mechanism $\cdot$ Influencing factors

\section{Introduction}

Austenitic stainless steel is widely used in many fields such as industry and commerce because of its good corrosion resistance [1]. Because of its poor thermal conductivity, severe work hardening, prone to buildup, high toughness, and ductility, it is classified as a difficult-to-process material [2-4]. As the most common austenitic stainless steel, AISI304 often encounters problems such as high cutting temperature, severe tool wear, and poor surface finish during processing. With the continuous improvement of requirements for diversified functions, the requirements for the surface quality and processing accuracy of parts are getting higher and higher, and it is difficult or impossible to meet the processing requirements with traditional cutting methods. To this end, people are constantly innovating on the road of exploring new cutting methods and put forward a series of non-conventional processing methods such as electric spark processing, laser-assisted processing. Studies have shown that applying ultrasound on the basis of traditional cutting can cause the tool tip or 
workpiece to vibrate at high frequencies and generate periodic motion trajectories during cutting, thereby achieving complete separation between the tool and the chip. This change in the cutting principle completely changed the method of reducing the cutting force by changing the cutting parameters or the sharpness of the tool in ordinary cutting, reducing the cutting temperature and tool wear, improving the surface quality and machining accuracy, especially in difficult-to-machine materials.

Ultrasonic vibration-assisted turning (UVT) can be divided into one-dimensional ultrasonic vibration-assisted turning (1D-UVT), ultrasonic elliptical vibration-assisted turning (UEVT), and three-excitation ultrasonic spatial vibration-assisted turning (3D-USVT) according to the vibration trajectory of the tool tip. 1D-UVT is to apply vibration in the cutting speed direction or feed direction on the basis of traditional cutting, so as to realize the discontinuous cutting of the tool and workpiece. Skelton [5] used 1D-UVT to cut low-carbon steel. The results show that compared with CT, 1D-UVT has a significant improvement in the surface finish of the machined surface at low cutting speed, but the improvement effect is not obvious at high cutting speed and believed that this phenomenon is caused by 1D-UVT reducing the generation of built-up tumors. Celaya et al. [6] used 1D-UVT cutting AISI1045 with vibration in the direction of the feed. Unlike literature [5], the surface finish of the machined surface increases with the increase of cutting speed, which is believed to be due to plastic side flow and the reduction of tool adhesion, and further shows that under different cutting parameters, the surface roughness obtained by 1D-UVT is lower than that of CT, and the surface quality is improved by more than 60\%. Luis et al. [7] applied vibrations in both directions of cutting speed and feed and studied the influence of 1D-UVT in different directions of vibration on surface roughness. The experimental results showed that, compared with CT, the 1D-UVT with vibration in the direction of cutting speed can obtain a lower surface roughness, the surface roughness obtained by the 1D-UVT with vibration in the direction of the feed is only slightly reduced. Babitsky et al. [8-9] cut aerospatial materials (nickel-based alloy C263 and Inconel718) by 1D-UVT with vibration in the feed direction. The results showed that compared with CT, the surface quality obtained by 1D-UVT has been greatly improved. Nath et al. [10] cut the Inconel718 by 1D-UVT with vibration in the direction of the cutting speed. The results show that the surface quality obtained by the 1D-UVT was improved by $75 \%-85 \%$ compared to CT under the same cutting conditions. Xu et al. [11-12] analyzed the machining performance of 1D-UVT and CT by machining AISI304 and Inconel718 and discussed the changing trend of surface roughness under different cutting parameters. The experimental results show that the surface roughness after ultrasonic vibration is significantly reduced in all cases. This experimental result is the same as the conclusion of Bai et al. [13].

According to the working principle of 1D-UVT, it can be found that the tool flank never separates from the machined surface. The high-frequency reciprocating vibration not only causes secondary plowing on the machined surface but also may cause the tool to peel off due to the alternating tension and compression stress. In order to overcome this phenomenon, Shamoto et al. [14] proposed a new cutting method, namely the ultrasonic elliptical vibration-assisted turning (UEVT), which 
applies vibration to the cutting edge simultaneously in the cutting speed direction and the chip outflow direction, so the cutting edge has an elliptical trajectory in a single movement cycle. Zhang et al. [15] believe that the UEVT cutting path is affected by vibration amplitude, tool geometry, cutting speed, and phase difference. Moriwaki et al. [16] compared the cutting performance of CT, 1D-UVT, and UEVT through experiments, and the results showed that UEVT can not only reduce the chip thickness and width but also improve the shape accuracy of the workpiece. In addition, UEVT also has the characteristics of suppressing the generation of burrs [17], reducing tool wear [18], increasing the critical cutting thickness [19], improving the machining accuracy of parts, and the stability of the workpiece system [20], etc. Zhang et al. [21] explored the relationship between cutting force, tool wear, surface roughness, chip formation, and cutting parameters (depth of cut, feed, and cutting speed) when cutting the end face of hardened steel with UEVT through experimental means. The results show that the cutting speed has the greatest impact, and the experimental results further prove that UEVT has good surface integrity. He et al. [22] analyzed the stress changes in the cutting area of the workpiece by UEVT in a single cutting cycle through finite element simulation, and the results showed that the tool plowing and unloading occurred in a cutting cycle. The stress generated when the cutting chips are not separated is significantly higher than the stress during separation. Compared with CT, UEVC has obvious stress changes, and the average stress produced is much smaller than $\mathrm{CT}$. The literature also further confirms that UEVT has a smaller cutting force and surface roughness. From the perspective of tribological performance, Mustapha et al. [23] studied the changing trend of the surface function parameters of $\mathrm{CT}$ and UEVT under different processing parameters (vibration amplitude, cutting speed, feed, and depth of cut). The results show that, compared with CT, the UEVT process can process workpieces with a higher surface function index. Due to the shortcomings of resonant UEVT, such as the elliptical trajectory shape and size are small and single, the device has kinematic coupling, and the piezoelectric ceramic sheet generates severe heat during operation, Ahn et al. [24] designed a non-resonant UEVT that directly drives the tool vibration through the low-frequency vibration of the piezoelectric ceramic sheet. The research results show that this method has excellent cutting performance. Kim et al. [25-27] also studied the non-resonant UEVT and realized the control of the cutting trajectory by changing the system parameters of the UEVT. At the same time, they deduced the relationship formula of the influencing factors of UEVT surface roughness. The relationship between the contact ratio and the speed ratio of the tool and the workpiece was studied. It is believed that the higher the contact ratio, the more obvious the reduction of cutting force. It is observed through experiments that compared with CT, the non-resonant UEVT has thinner chips, larger shear angles, and reduced burrs during cutting, which improves the shape accuracy of the workpiece, but the surface of the workpiece in contact with the tool has obvious chatter marks, and the surface finish is significantly lower than that of CT. This phenomenon corresponds to the literature [28]. But this phenomenon does not appear in the literature [29]. Through the research on chip performance, it is found that compared with CT, the non-resonant UEVT 
produces thinner chips and the shear angle becomes larger. The research results show that the non-resonant UEVT can suppress the generation of burrs and improve the shape accuracy of the workpiece. The higher the frequency, the more obvious the cutting characteristics of UEVT and the higher the surface accuracy of the workpiece.

With the continuous development of ultrasonic ellipse vibration-assisted turning technology, people began to study three-dimensional ellipse vibration-assisted turning(3D-EVT). Compared with UEVT, 3D-EVT can make the tool realize any elliptical trajectory in three-dimensional spatial [30], which is more suitable for processing free-form surfaces [31]. The current research on 3D-EVT is mainly based on the structural design and output stability of the cutting system [32]. Among them, Shamoto et al. [31] established a three-degree-of-freedom ultrasonic vibration system by coupling two bending vibrations and one longitudinal resonance and obtained successfully flat and spherical mirrors in quenched die steel. Kurniawan et al. [33-34] analyzed the transducer through finite element technology and believed that three-dimensional elliptical motion at ultrasonic frequencies could be achieved by changing the size of the transducer, and established a 3D-EVT geometric model of the surface topography. The validity of the model is verified by experiments. By cutting AL6160, it is found that the surface roughness obtained by 3D-EVT is significantly lower than that of CT. Sajjady et al. [35] established a micro-topography generation model of the workpiece, which can simulate the micro-topography of 1D-UVT, UEVT, and 3D-EVT, and the correctness of the model was verified by experiments. The experimental results are consistent with those observed by Kurniawan et al, but the surface roughness obtained by 3D-EVT is larger than that obtained by 1D-UVT and UEVT. In terms of non-resonant 3D-EVT, Lin et al. [36-37] designed and studied the non-resonant 3D-EVT turning system, and studied the performance of the system through experiments. The experimental results show that compared with CT, UEVT, non-resonant 3D-EVT can not only reduce the surface roughness of the workpiece but also improve the integrity of the workpiece surface. Zhu et al. [38-39] studied the non-resonant 3D-EVT based on the EFCS system, the tool rotated with the lathe spindle and applied three-dimensional vibration on the workpiece or tool. This realizes the micro-nano structure surface of the workpiece. Compared with other non-resonant 3D-EVT systems, this method has the disadvantages of large volume and complex structure. Due to the initial stress, the nonlinearity of the material, and the large deformation, the non-resonant 3D-EVT system generally has nonlinearity and uncertainty. In order to solve this problem, Lin et al. [40-41] combined the non-resonant 3D-EVT system with robust adaptive fuzzy control technology to improve the stability of the system. Compared with the ordinary non-resonant 3D-EVT system, the surface roughness obtained by the improved 3D-USVT system is reduced by $20 \% \sim 32 \%$.

the current research on the output shape of the 3D-EVT tool nose vibration trajectory is mainly based on the spatial ellipse, and there is almost no research on the other vibration output shapes of the tool. To better explain the impact of the tooltip vibration trajectory on the surface quality of the workpiece, this paper proposes a novel resonant three-excitation ultrasonic spatial vibration-assisted turning(3D-USVT) 
system driven by three excitations. The nose of the tool can generate complex, stable, and closed vibration trajectories in a single cycle. The vibration trajectory keeps the chip flow angle in a changing state during the cutting process, which facilitates the discharge of chips and improves the surface quality of the workpiece.

\section{3D-USVT cutting system}

The 3D-USVT applies excitation vibration in three directions at the same time, so that the vibration trajectory of the tool tip in the three-dimensional spatial is a stable and closed curve. Fig. 1 shows the tool tip vibration trajectory of the 3D-USVT under different frequency ratios. It can be seen that when the vibration frequencies in the three directions are not equal, as the frequency ratio increases, within one cycle, the more complex the vibration trajectory of the tool tip. However, the complex shape of the vibration trajectory causes too much vibration of the tool, which will not only produce large alternating stress and aggravate the wear of the tool, but also cause tool chatter and deteriorate the surface quality of the workpiece. As the excitation frequency increases, not only does the stability of the entire system decrease, it is also difficult to guarantee the output power of the transducer with the existing manufacturing technology. When vibration is applied in three different directions at the same time, the resulting vibration displacement will cause a certain coupling phenomenon between each other, Studies [42] have shown that the orthogonal output structure can not only effectively reduce the impact caused by the different directions of vibration displacement, but also make it easier to combine and output vibration displacements in different directions. When the plane where the tool vibration path is located is coplanar with the plane where the chip discharge direction is located, a higher surface quality can be obtained. The chip discharge direction is related to the workpiece material. To improve the processing performance of different workpiece materials, this paper uses the tool tip vibration trajectory in Fig. 1 (b) as the output trajectory of 3D-USVT.

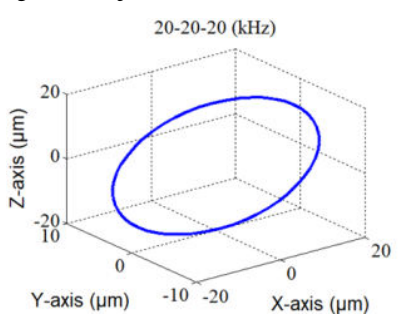

(a) Frequency ratio 1:1:1

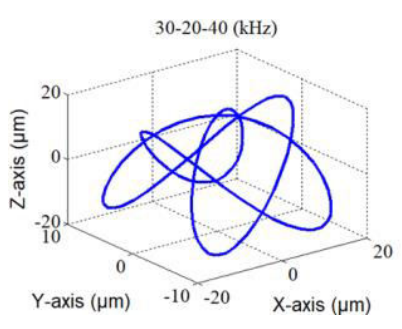

(d) Frequency ratio 3:2:4

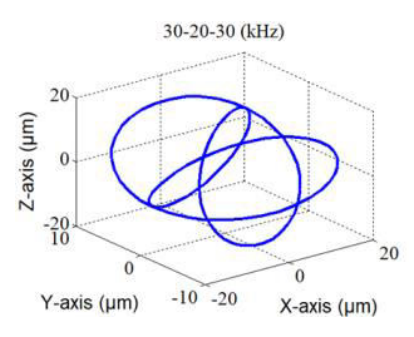

(b) Frequency ratio 3:2:3

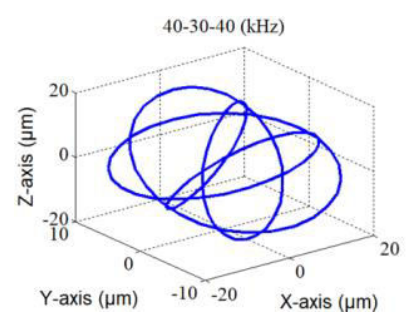

(e) Frequency ratio 4:3:4

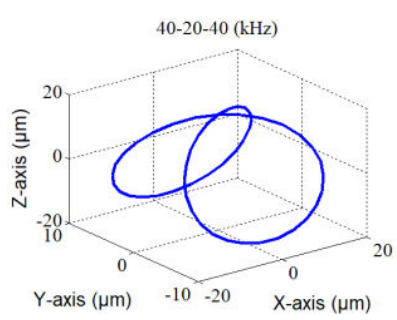

(c) Frequency ratio 2:1:2

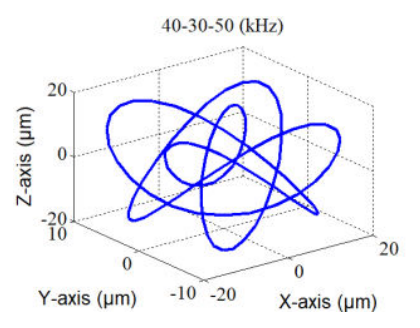

(f) Frequency ratio 4:3:5

Fig.1 Tool tip vibration trajectory of 3D-USVT under different frequency ratios Therefore, the 3D-USVT studied in this paper adopts a quadrature output structure. 
Simultaneously apply two excitations of excitation frequency of $30 \mathrm{kHz}$ and a phase difference of $90^{\circ}$ to the 3D-USVT in the horizontal direction, and apply a single excitation with an excitation frequency of $20 \mathrm{kHz}$ and an initial phase of zero to the 3D-USVT in the vertical direction. Three excitations work together vertically to make the cutting edge of the tool produce a simple, stable, and closed vibration trajectory in the three-dimensional spatial.

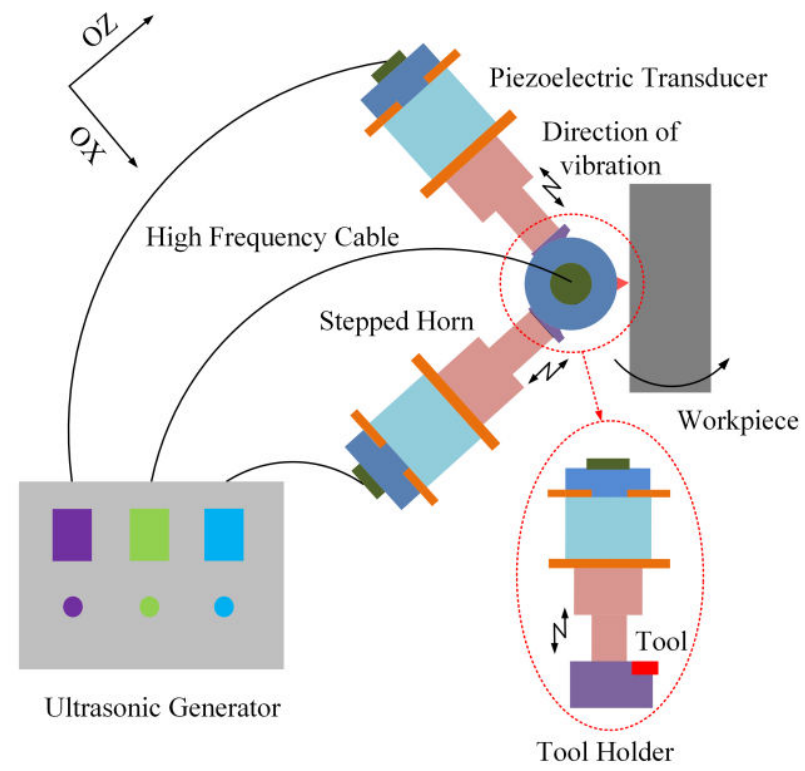

Fig. 2 Schematic diagram of 3D-USVT

The 3D-USVT studied in this paper directly applies the vibration displacement to the tool holder through the luffing system so that the tool tip produces a three-dimensional trajectory. To study the effect of ultrasonic vibration on the surface roughness of the workpiece, the tool holder coordinates need to be established. In order to facilitate the installation, the 3D-USVT studied in this paper is equipped with two excitation devices at $90^{\circ}$ in the horizontal direction. The vibration exerted on the tool does not directly act on the depth of cut direction and the feed direction but acts in a direction that rotates $\pi / 4$ along the main coordinate system $\mathrm{O}_{2} \mathrm{Y}_{2}$ of the machine tool. First, set the tool fillet center as the origin of the assumed working plane reference system OXYZ, and define the intersection line formed by the back plane, base plane and the assumed working plane, which are perpendicular to each other, they are defined as the axis of OX, OY, OZ, converting the assumed work plane reference system $\mathrm{OXYZ}$ to the tool-holder coordinate system $\mathrm{O}_{1} \mathrm{X}_{1} \mathrm{Y}_{1} \mathrm{Z}_{1}$ by coordinate transformation. When the 3D-USVT system is working, the coordinate of any point of the tool can be expressed in the tool holder coordinate system as

$$
\left[\begin{array}{l}
X_{1} \\
Y_{1} \\
Z_{1}
\end{array}\right]=R_{x}\left(-\lambda_{s}\right) \cdot R_{y}\left(-k_{r}\right) \cdot R_{z}\left(-\alpha_{0}\right)\left[\begin{array}{l}
X \\
Y \\
Z
\end{array}\right]+\left[\begin{array}{c}
a \cdot \sin \left(2 \pi f_{1} t\right) \\
b \cdot \cos \left(2 \pi f_{2} t\right) \\
c \cdot \sin \left(2 \pi f_{1} t\right)
\end{array}\right]
$$

where $\lambda_{s}, k_{r}$ and $\alpha_{0}$ respectively represent the cutting edge inclination angle, entering angle and rake angle of the tool. $a, b$, and $c$ respectively represent the vibration amplitudes in the three vibration directions, $f_{1}, f_{2}$ represents the vibration 
frequencies in the three directions, and $f_{1} / f_{2}$ are rational numbers.

Assuming that the origin of the machine's main coordinate system is located on the axis of the processed bar, and the motion path of the tool in the machine's main coordinate system $\mathrm{O}_{2} \mathrm{X}_{2} \mathrm{Y}_{2} \mathrm{Z}_{2}$ can be expressed as

$$
\left[\begin{array}{l}
X_{2} \\
Y_{2} \\
Z_{2}
\end{array}\right]=R_{y}\left(\frac{\pi}{4}\right) \cdot\left[\begin{array}{l}
X_{1} \\
Y_{1} \\
Z_{1}
\end{array}\right]+\left(\begin{array}{c}
S-D O C \\
0 \\
0
\end{array}\right)+\left(\begin{array}{c}
0 \\
v t \\
0
\end{array}\right)+\left(\begin{array}{c}
0 \\
0 \\
L-f f_{r} t
\end{array}\right)
$$

where $L$ is the total length of the bar, $f$ is the feed during normal cutting, $f_{r}$ is the rotation frequency of the workpiece, $D O C$ is the depth of cut, $S=r_{\varepsilon}+R$ represents the distance between the center of the tool tip arc and the axis of the workpiece after tool setting, $r_{\varepsilon}$ is the corner radius, $R$ is the initial radius of the workpiece, and $v$ is the cutting speed during processing.

\section{Experiment setup and steps}

The machine tool selected for the experimental platform in this paper is the general lathe CA6140, with 304 stainless steel as the cutting object, the diameter is $35 \mathrm{~mm}$. The tool is a CCMT120404-HMP cemented carbide tool with a tip fillet radius of $0.4 \mathrm{~mm}$. In order to produce a closed Lissajous vibration trajectory of the tool in 3D spatial, the system vibration generator is adjusted to realize the 3D-USVT driven at different vibration frequencies. The 3D-USVT is fixedly installed on the slide box of the lathe by bolts. During the machining process, the movement and cutting of the tool in the direction of cutting speed and feed are realized by spindle rotation.

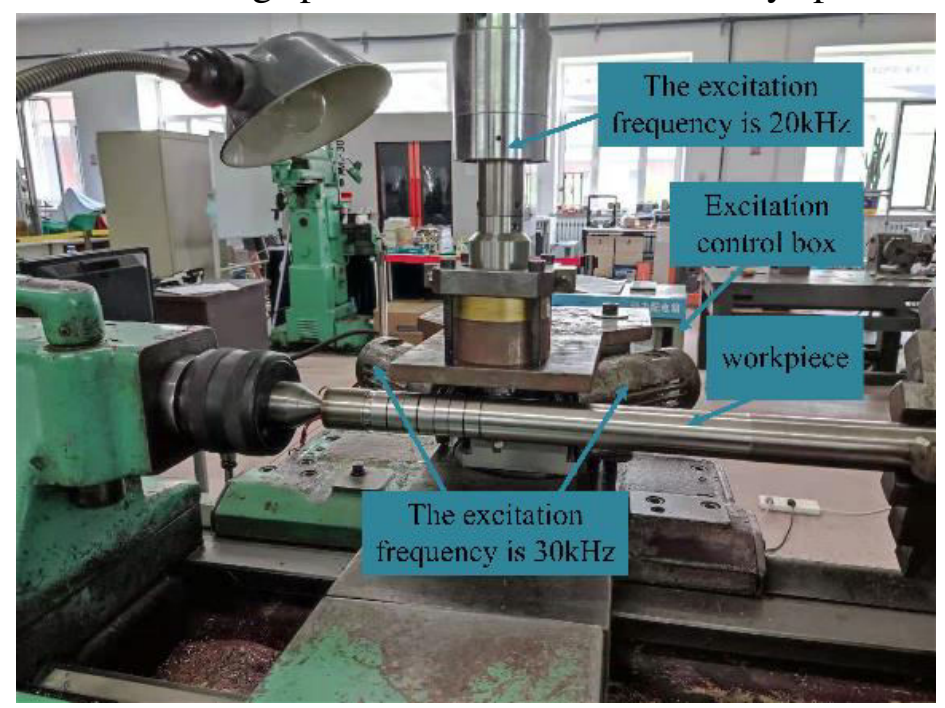

Fig. 3 3D-USVT system

To verify the processing feasibility of the 3D-USVT system, the vibration textures of CT, 1D-UVT, UEVT, and 3D-USVT were compared. Under the same cutting parameters (cutting speed, depth of the cut, and feed), CT, 1D-UVT, UEVT, and 3D-USVT are used to cut 304 stainless steel, respectively. After the processing is completed, a micromeasure 3D profiler and digital optical microscope were used to observe the macroscopic and microscopic topography of the workpiece surface, as shown in Fig. 4 and Fig. 5. 


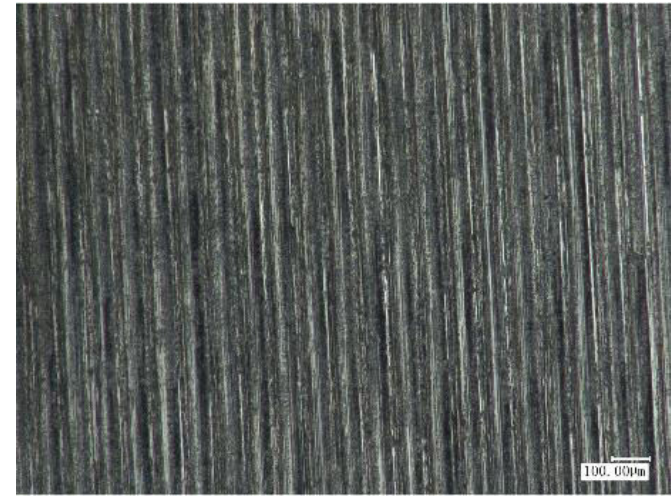

(a) CT surface topography

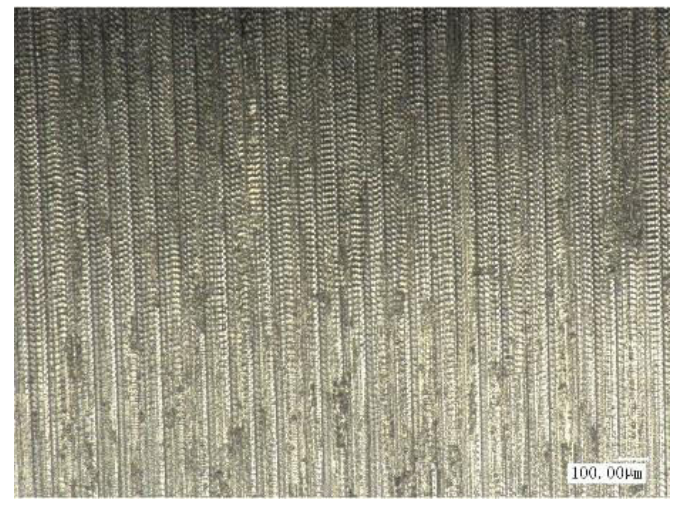

(c) UEVT surface topography

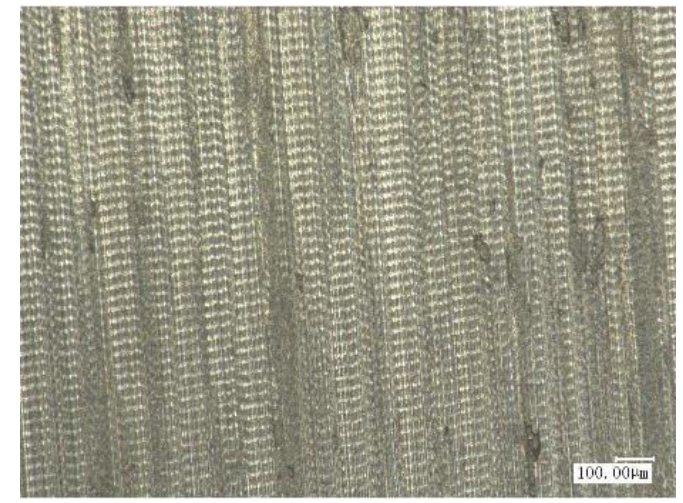

(b) 1D-UVT surface topography

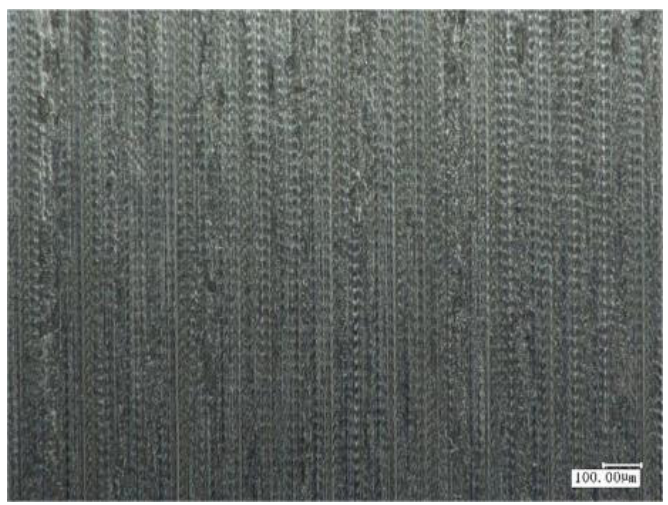

(d) 3D-USVT surface topography

Fig. 4 Comparison of the macro-morphology of the workpiece surface under different cutting methods

Under the same cutting conditions, when cutting with the CT method, brittle fracture occurs on the surface of the workpiece due to the mutual extrusion and friction between the tool and the workpiece during the cutting process, and the processed surface is composed of linear grooves and ridges of varying depths, which are caused by cutting feed and tool profile. During this period, plastic deformation of the workpiece and tool friction is caused by the long-term non-separation and extrusion between the tool and the workpiece material and the surface of the workpiece also shows irregular cracks, material peeling, scratches, and build-up of debris sticking, even scaly thorns. The machined surface produced by 1D-UVT is composed of vibration tracks and tool feed marks that are similar in depth and uniformly distributed like tire indentation. Due to the cutting characteristics of 1D-UVT, the machined surface is prone to scratches, which destroy the integrity of the machined surface. However, UEVT produces a regularly distributed vibration texture with intermittent concave characteristics on the machined surface. It can be seen from Fig. 4(c) that the machined surface produced by this cutting method is prone to "feeding ridges" in the feed direction. The machined surface produced by 3D-USVT forms an intermittent and evenly distributed vibration texture like tooth marks in the direction of the tool path. Compared with UEVT, this cutting method is also prone to a "feeding ridge" in the feed direction, but the "feed ridge" is not distinct. Regardless of the linear grooves and ridges produced by common turning (CT) or the 
vibration lines of different shapes engender by ultrasonic vibration-assisted turning (UVT), these will cause the surface quality of the workpiece to deteriorate.

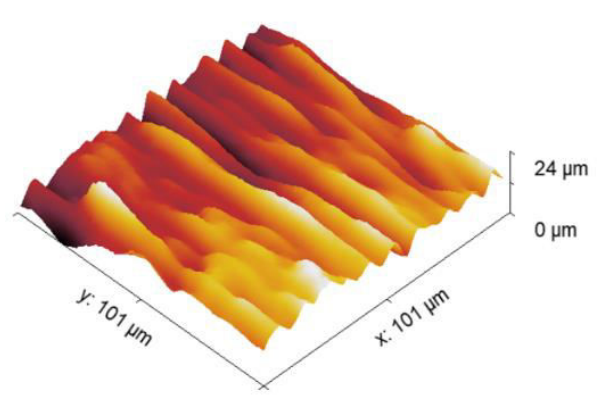

(a) CT three-dimensional topography

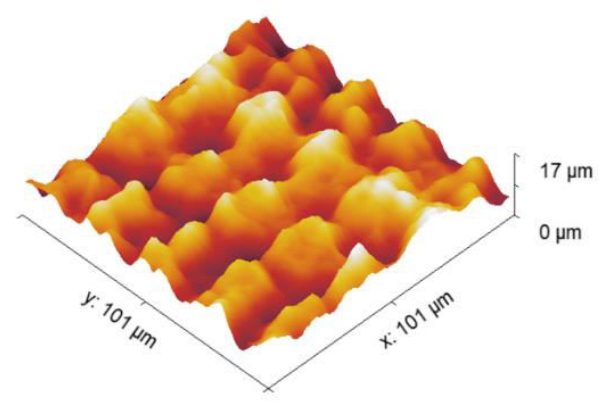

(c) UEVT three-dimensional topography

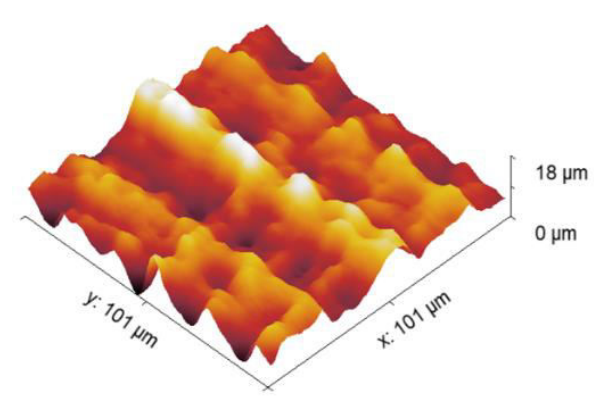

(b) 1D-UVT three-dimensional topography

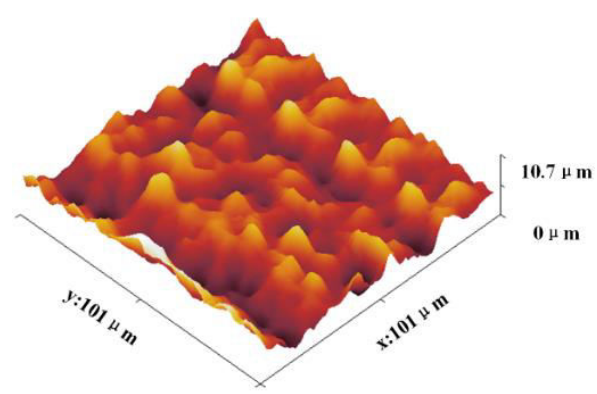

(d) 3D-USVT three-dimensional topography

Fig. 5 The surface micro-morphology of the workpiece under different cutting methods

It can be seen from Fig. 5 that under the same cutting parameters, the residual geometric height of the machined surface produced by the CT method is significantly higher than that of other methods, indicating that the ultrasonic vibration-assisted cutting method can significantly improve the quality of the machined surface of the workpiece. Compared with 1D-UVT and UEVT, the residual geometric height of the workpiece surface obtained by 3D-USVT is the smallest, indicating that the 3D-USVT method has good processing performance. 


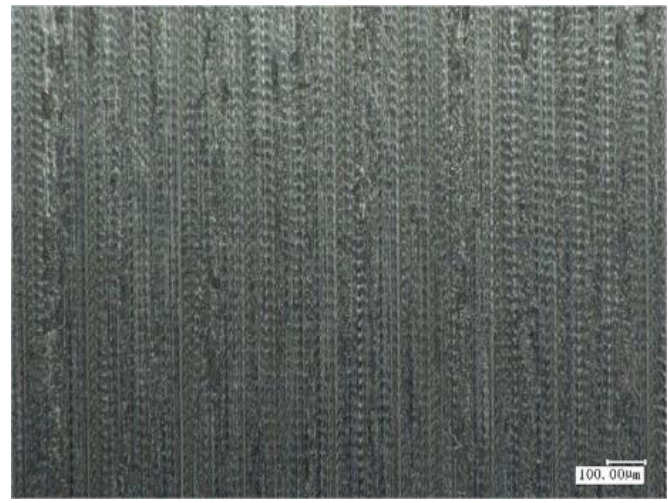

(a) $b=8 \mu \mathrm{m}, c=3 \mu \mathrm{m}, \mathrm{a}=5 \mu \mathrm{m}$

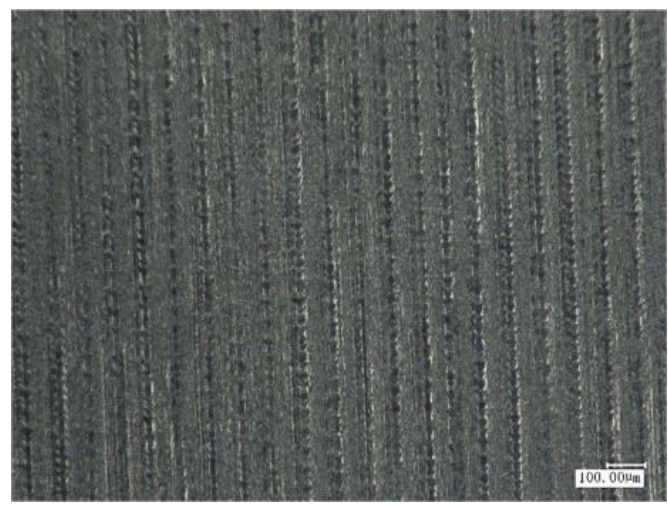

(c) $b=12 \mu \mathrm{m}, c=6 \mu \mathrm{m}, \mathrm{a}=6 \mu \mathrm{m}$

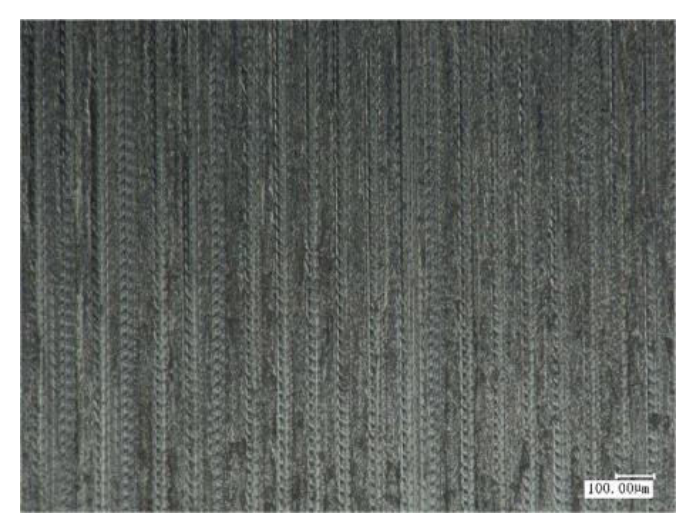

(b) $b=10 \mu \mathrm{m}, c=5 \mu \mathrm{m}, \mathrm{a}=6 \mu \mathrm{m}$

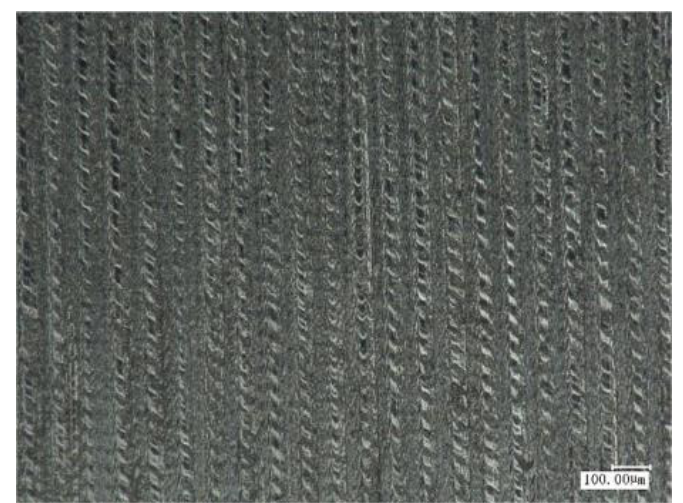

(d) $b=12 \mu \mathrm{m}, c=9 \mu \mathrm{m}, a=8 \mu \mathrm{m}$

Fig. 6 The macro-morphology of the workpiece surface under different amplitudes

It can be seen from Fig. 6 that as the amplitude continues to increase, the processed surface becomes increasingly rough. With the increase of $\mathrm{X}$-axis amplitude and Z-axis amplitude, the intermittent pits produced by the cutter in the depth of cut direction are more obvious, and the plow slip marks produced by the cutter in the feed direction become more and more obvious. From this, the surface quality of the workpiece obtained by the 3D-USVT method is related to the magnitude of the vibration amplitude. In addition, the surface quality of the workpiece is also related to the cutting speed $(v)$, feed $(f)$, and depth of cut $\left(a_{p}\right)$ [44].

\subsection{Response surface analysis of vibration amplitude}

To better analyze the impact of vibration amplitude in three directions on the surface roughness of 3D-USVT during the cutting process, this paper adopts the BBD method and takes the amplitude in the three directions as the research factors, obtaining the influence of different amplitudes on surface roughness. Table 1 shows the range of amplitude in three directions.

Table 1 Selection of the 3D-USVT amplitude parameters

\begin{tabular}{cccc}
\hline Level & $\begin{array}{c}\text { Y direction vibration } \\
\text { amplitude }(\mu \mathrm{m})\end{array}$ & $\begin{array}{c}\text { X direction vibration } \\
\text { amplitude }(\mu \mathrm{m})\end{array}$ & $\begin{array}{c}\text { Z direction vibration } \\
\text { amplitude }(\mu \mathrm{m})\end{array}$ \\
\hline-1 & 5 & 3 & 3 \\
0 & 10 & 6 & 6 \\
1 & 15 & 9 & 9 \\
\hline
\end{tabular}


To describe accurately the surface roughness of the workpiece, this paper uses the three-dimensional surface roughness parameter $\mathrm{Sa}$ as the standard for evaluating turning processing parameters [45-46]. Take the amplitude of 3D-USVT in the X, Y, and $\mathrm{Z}$ directions as independent variables, and $\mathrm{Sa}$ as the response value for response surface experiments. The experimental plan and results are shown in Table 2. To ensure the accuracy of the experimental data, three repeatability tests were done for each group of data. Three points are selected on the surface of the workpiece obtained in each experiment to measure the three-dimensional profile of the surface, and the average of the measurement results is taken as the test data of each set of the Sa value.

Table 2 Response surface analysis experimental plan and experimental results

\begin{tabular}{ccccc}
\hline Serial number & $\begin{array}{c}\text { Y direction } \\
\text { vibration } \\
\text { amplitude }(\text { A })\end{array}$ & $\begin{array}{c}\text { X direction } \\
\text { vibration } \\
\text { amplitude } \\
(\text { B })\end{array}$ & $\begin{array}{c}\text { Z direction } \\
\text { vibration } \\
\text { amplitude } \\
(\text { C })\end{array}$ & Sa \\
\hline 1 & 10 & 3 & 3 & 1.302 \\
2 & 5 & 3 & 6 & 1.591 \\
3 & 10 & 6 & 6 & 1.459 \\
4 & 10 & 9 & 3 & 1.513 \\
5 & 5 & 9 & 6 & 1.829 \\
6 & 15 & 6 & 9 & 1.491 \\
7 & 15 & 6 & 3 & 1.275 \\
8 & 10 & 3 & 9 & 1.508 \\
9 & 10 & 6 & 6 & 1.486 \\
10 & 5 & 6 & 9 & 1.673 \\
11 & 10 & 6 & 6 & 1.502 \\
12 & 10 & 6 & 6 & 1.536 \\
13 & 15 & 9 & 6 & 1.682 \\
14 & 10 & 9 & 9 & 1.684 \\
15 & 15 & 3 & 6 & 1.412 \\
16 & 5 & 6 & 3 & 1.427 \\
17 & 10 & 6 & 6 & 1.493 \\
\hline
\end{tabular}

Table 3 Analysis of variance of different Sa models

\begin{tabular}{cccccccc}
\hline $\begin{array}{c}\text { Process } \\
\text { order }\end{array}$ & Model & Lack-of-fit & $\begin{array}{c}\text { R-Squar } \\
\text { ed }\end{array}$ & $\begin{array}{c}\text { R-Squar } \\
\text { ed (adj) }\end{array}$ & $\begin{array}{c}\text { Pred } \\
\text { R-Squar } \\
\text { e }\end{array}$ & $\begin{array}{c}\text { Adeq } \\
\text { precision }\end{array}$ & $\begin{array}{c}\text { C.V. } \\
\%\end{array}$ \\
\hline Liner & Significant & significant & 0.7772 & 0.7257 & 0.5537 & 12.218 & 4.81 \\
2FI & Significant & $\begin{array}{c}\text { Significant } \\
\text { Not }\end{array}$ & 0.7797 & 0.6475 & -0.0481 & 8.147 & 5.45 \\
Quadratic & Significant & $\begin{array}{c}0.9813 \\
\text { significant }\end{array}$ & 0.9571 & 0.8439 & 24.289 & 1.90 \\
\hline
\end{tabular}

Through the analysis and comparison of three different models of $\mathrm{Sa}$, it can be seen from Table 3 that the model and lack-of-fit of the three models only meet the requirements of the Quadratic model. The R-Sq value and R-Sq (adj) of the Quadratic model are relatively high, indicating that the model has a high degree of fit with the 
experimental data, and the accuracy of predicting the response value is also high; the $\mathrm{R}-\mathrm{Sq}$ value of the Quadratic model is close to the R-Sq (adj) value, indicating that the reliability of the model is higher; the signal-to-noise ratio and CV\% value of the Quadratic model are the maximum and minimum values in the three models, respectively, which shows the accuracy and reliability of the experimental data.

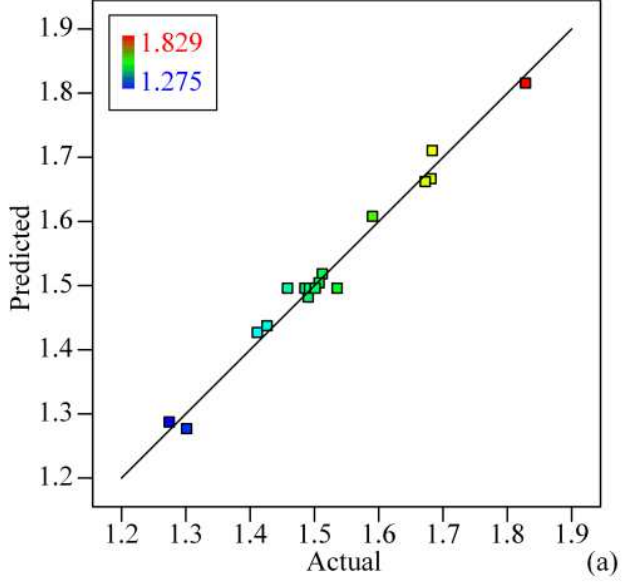

(a) Experimental results and predicted values

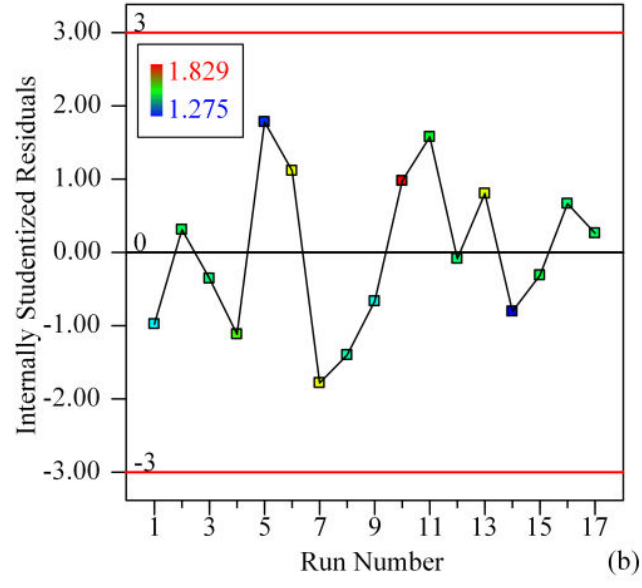

(b) Internal standard residual

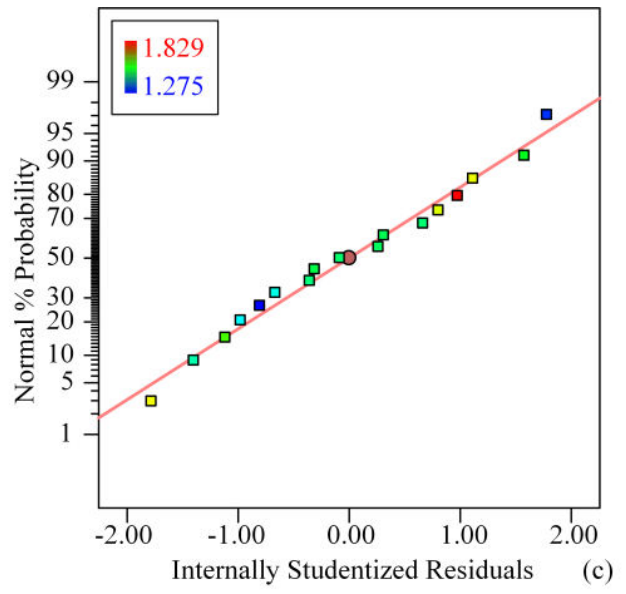

(c) Normal probability

Fig. 7 Applicability of Sa model

Fig. 7(a) shows that the experimental values are concentrated on both sides of the predicted value, reflecting the consistency between the experimental values and the predicted values. Fig. 7(b) shows that the residual points are concentrated in the horizontal band formed by -3 and +3 , it shows the validity of the selected Quadratic model. Fig. 7(c) shows that the experimental points obey the normal distribution, which verifies the reliability of the Quadratic model. 

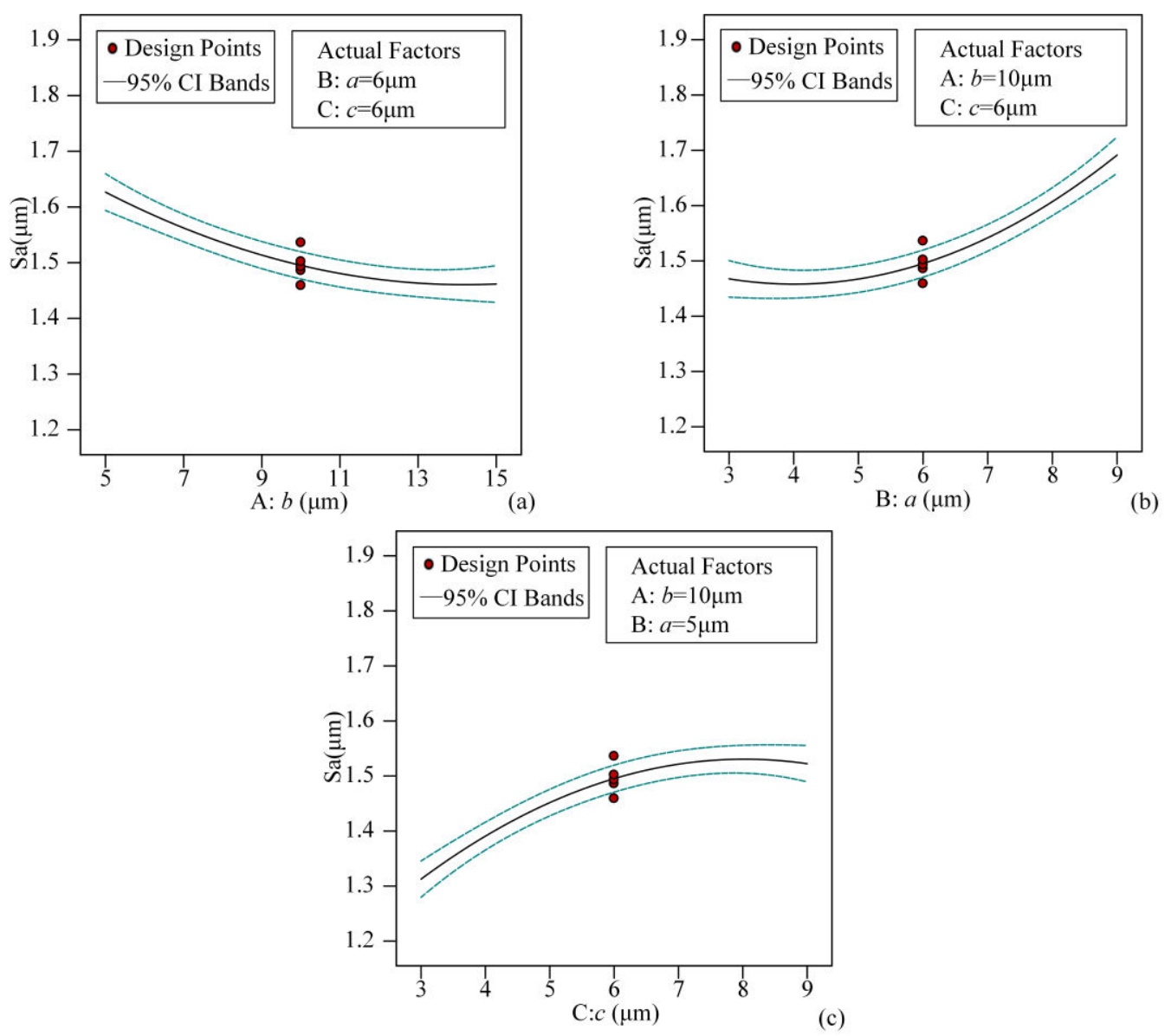

Fig. 8 Effect diagram of a single factor of Sa

The three mutually perpendicular excitations work together to realize the complete separation between the tool, the chip, and the workpiece, which not only shortens the net cutting time for the tool to participate in the cutting but also causes cracks on the workpiece to be processed due to the extreme instantaneous acceleration of the tool. The cutting force is reduced, the tool wear is reduced and the system stability is improved to reduce Sa. Fig. 8(a) shows that when the Y direction amplitude is greater than $11 \mu \mathrm{m}$, the decreasing trend of Sa gradually becomes slower. When the $\mathrm{Y}$ direction vibration amplitude is equal to $15 \mu \mathrm{m}$, the Sa value appears to rise. The reason for this phenomenon, probably because as the amplitude increases, the mechanical impact of the tool becomes larger, and the excessive impact force causes additional vibration of the tool, which reduces the stability of the 3D-USVT system; the material removal area of the workpiece increases, the cutting temperature increases, and the momentary impact on the tool tip becomes larger. This not only aggravates tool wear, but also reduces the stability of the 3D-USVT, and eventually leads to the gradual deterioration of workpiece surface quality.

With the increase of the amplitude in the $\mathrm{X}$ direction, the tool produces a great instantaneous component acceleration in the feed direction, and the workpiece material is subjected to mechanical impact to produce cracks, which reduces the cutting force and the Sa value. At the same time, with the increase of the amplitude in the $\mathrm{X}$ direction, the tool generates deeper vibrations along the cutting path in the depth-of-cut direction, which increases the Sa value. The changing trends of the two 
types of Sa restrict each other. Therefore, when the X direction amplitude is less than $5 \mu \mathrm{m}$, Sa changes slowly under the constraints of these two changing trends, as shown in Fig. 8(b). When the $X$ direction amplitude is greater than $5 \mu \mathrm{m}$, the cutting path of the tool along the feed direction becomes wider, which improves the cutting accuracy and processing efficiency to a certain extent, but the mechanical impact of the tool becomes larger, and the excessive impact force not only causes the tool to produce additional vibration will aggravate the wear of the tool, reduce the stability of the system, and increase the value of Sa.

It can be seen from Fig. 8(c) that when the amplitude in the $\mathrm{Z}$ direction is less than $6 \mu \mathrm{m}$, the Sa value increases with the increase of the amplitude in the $\mathrm{Z}$ direction. This may be because with the increase of the amplitude in the $\mathrm{Z}$ direction, the vibration texture generated by the tool in the depth direction is more obvious, and the surface quality of the workpiece is worse. When the amplitude of the $\mathrm{Z}$ direction is greater than $6 \mu \mathrm{m}$, the increase of Sa begins to become flat. This may be because of the continuous increase in the amplitude of $\mathrm{Z}$ direction, the continuous overlap rate of the vibration trajectories of adjacent cutting paths is increased, which to a certain extent the height of the ridge generated on the machined surface of the workpiece in the previous cutting is reduced, and the increase of $\mathrm{Sa}$ is delayed.
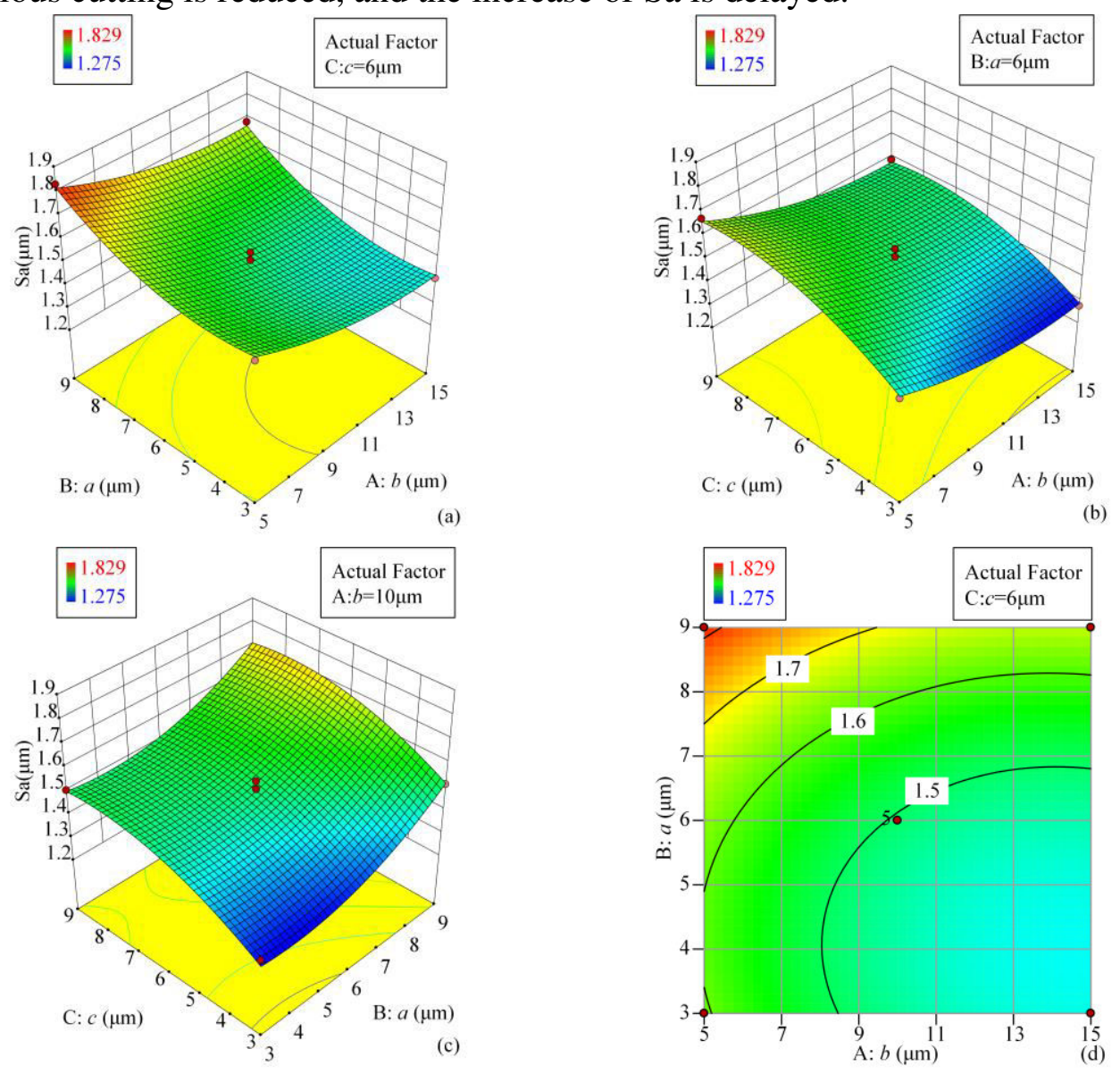

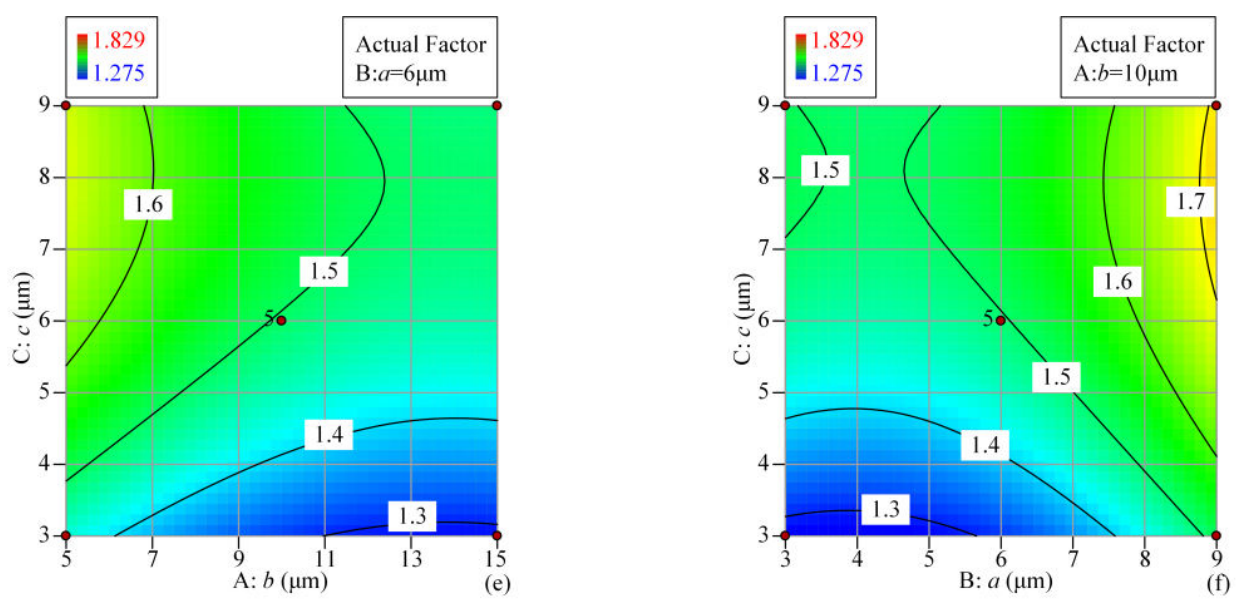

Fig. 9 Response surface plot and contour plot of SA under the action of vibration amplitude

Fig. 9 is the response surface plot and contour plot of Sa under different amplitudes. Fig. 9(a) can be seen that when the Y direction amplitude takes different values, the value of $\mathrm{Sa}$ increases nonlinearly with the increase of the $\mathrm{X}$ direction amplitude. Conversely, when the amplitude in the $\mathrm{X}$ direction takes different values, the value of Sa decreases nonlinearly with the increase in the amplitude in the Y direction. This shows that the interaction between the $\mathrm{Y}$ direction amplitude and the $\mathrm{X}$ direction amplitude is not significant. The change rate of $\mathrm{Sa}$ along with the $\mathrm{X}$ direction amplitude enlarge with the $\mathrm{Y}$ direction amplitude increase, and when the Y direction amplitude is equal to $14 \mu \mathrm{m} \sim 15 \mu \mathrm{m}$, the change rate of Sa reaches the maximum, and the $\mathrm{Y}$ direction amplitude is equal to $5 \mu \mathrm{m} \sim 6 \mu \mathrm{m}$, the change rate of Sa reaches the minimum; the change rate of Sa along with the Y direction amplitude decreases as the $\mathrm{X}$ direction amplitude increases, and when the $\mathrm{X}$ direction amplitude is equal to $3 \mu \mathrm{m} \sim 4 \mu \mathrm{m}$, the change rate of Sa reaches the maximum, and the $\mathrm{X}$ direction amplitude is equal to $8 \mu \mathrm{m} \sim 9 \mu \mathrm{m}$, the change rate of Sa reaches the minimum.

Fig. 9(b) shows that when the $\mathrm{Y}$ direction amplitude takes different values, the value of $\mathrm{Sa}$ increases nonlinearly with the increase of the $\mathrm{Z}$ direction amplitude. On the contrary, when the $\mathrm{Z}$ direction amplitude takes different values, the value of $\mathrm{Sa}$ increases with the increase in $\mathrm{Y}$ direction amplitude decreases non-linearly, which shows that the interaction between $\mathrm{Y}$ direction amplitude and $\mathrm{Z}$ direction amplitude is not significant. The rate of change of the amplitude of $\mathrm{Sa}$ along the $\mathrm{Z}$ direction decreases with the increase of the amplitude of the $\mathrm{Y}$ direction, and when the amplitude of the Y direction is equal to $5 \mu \mathrm{m} \sim 6 \mu \mathrm{m}$, the rate of change of Sa reaches the maximum, and the amplitude of the $\mathrm{Y}$ direction is equal to $14 \mu \mathrm{m} \sim 15 \mu \mathrm{m}$, the change rate of Sa reaches the minimum; the change rate of Sa along with the $\mathrm{Y}$ direction amplitude enlarge with the increase of the $\mathrm{Z}$ direction amplitude, and when the $\mathrm{Z}$ direction amplitude is equal to $8 \mu \mathrm{m} \sim 9 \mu \mathrm{m}$, the change rate of Sa reaches the maximum, and the $\mathrm{Z}$ direction amplitude is equal to $3 \mu \mathrm{m} \sim 4 \mu \mathrm{m}$, the change rate of $\mathrm{Sa}$ reaches the minimum.

Fig. 9(c) can be seen that when the amplitude of the $\mathrm{Z}$ direction is different, the value of $S a$ increases non-linear with the increase of the amplitude of the $\mathrm{X}$ direction; when the amplitude of the $\mathrm{X}$ direction takes different values, the value of Sa increases 
non-linear with the increase of the amplitude of the $\mathrm{Z}$ direction, which shows that the interaction between the $\mathrm{X}$ direction amplitude and the $\mathrm{Z}$ direction amplitude is not significant. The change rate of $\mathrm{Sa}$ along with the $\mathrm{X}$ direction amplitude decrease as the $\mathrm{Z}$ direction amplitude increases, and when the $\mathrm{Z}$ direction amplitude is equal to $3 \mu \mathrm{m} \sim 4 \mu \mathrm{m}$, the change rate of Sa reaches the maximum, and the $\mathrm{Z}$ direction amplitude is equal to $8 \mu \mathrm{m} \sim 9 \mu \mathrm{m}$, the change rate of Sa reaches the minimum; the change rate of $\mathrm{Sa}$ along with the $\mathrm{Z}$ direction amplitude decrease as the $\mathrm{X}$ direction amplitude increases, and when the $X$ direction amplitude is equal to $3 \mu \mathrm{m} \sim 5 \mu \mathrm{m}$, the change rate of Sa reaches the maximum, and the $X$ direction amplitude is equal to $8 \mu \mathrm{m} \sim 9 \mu \mathrm{m}$, the change rate of Sa reaches the minimum.

It can be seen from Figs. 9(a)、9(b), and 9(c) that when the X direction amplitude and the $\mathrm{Z}$ direction amplitude are smaller, and the $\mathrm{Y}$ direction amplitude is larger, smaller surface roughness can be obtained.

It can be seen from Fig. 9(d) and Fig. 9(f) that, compared to the Y direction amplitude and the $\mathrm{Z}$ direction amplitude, the $\mathrm{X}$ direction amplitude has a more significant impact on Sa; Fig. 9(e) shows that the amplitude of $\mathrm{Z}$ direction has a more significant effect on Sa than the amplitude of Y direction. It can be seen that the influence order of the amplitude in the three directions of 3D-USVT on the Sa value is: $\mathrm{X}$ direction amplitude $>\mathrm{Z}$ direction amplitude $>\mathrm{Y}$ direction amplitude.

In order to obtain the best response under the interaction of different factors, the target value of $\mathrm{Sa}$ is set to the minimum, and the factor is set to the unconstrained state. The obtained optimal vibration amplitude combination is shown in Table 4 .

Table 4 Optimal amplitude parameter values

\begin{tabular}{ccc}
\hline Y direction amplitude $(\mu \mathrm{m})$ & $\mathrm{X}$ direction amplitude $(\mu \mathrm{m})$ & $\mathrm{Z}$ direction amplitude $(\mu \mathrm{m})$ \\
\hline 14.13 & 3.74 & 3 \\
\hline
\end{tabular}

\subsection{Response surface analysis of cutting parameters}

In order to better explain the effect of the 3D-USVT on surface roughness, based on the optimized amplitude, the experiment also studied the influence of cutting parameters on Sa. The cutting speed $(v)$, depth of cut $\left(a_{p}\right)$, and feed $(f)$ of 3D-USVT are used as independent variables, and $\mathrm{Sa}$ is the response value for the response surface experiment. The level selection of cutting factors, experimental plan, and results are shown in Table 5 and Table 6 respectively.

Table 5 Selection of 3D-USVT cutting parameters

\begin{tabular}{cccc}
\hline Level & $v(\mathrm{r} / \mathrm{min})$ & $a_{p}(\mathrm{~mm})$ & $f(\mathrm{~mm})$ \\
\hline-1 & 100 & 0.05 & 0.08 \\
0 & 250 & 0.1 & 0.14 \\
1 & 400 & 0.15 & 0.2 \\
\hline
\end{tabular}

Table 6 Response surface methodology analysis experimental plan and experimental results

\begin{tabular}{ccccc}
\hline Serial number & $v(\mathrm{r} / \mathrm{min})$ & $a_{p}(\mathrm{~mm})$ & $f(\mathrm{~mm})$ & $\mathrm{Sa}$ \\
\hline 1 & 250 & 0.05 & 0.08 & 1.325 \\
2 & 250 & 0.05 & 0.2 & 1.973 \\
\hline
\end{tabular}




\begin{tabular}{ccccc}
\hline 3 & 250 & 0.1 & 0.14 & 1.653 \\
4 & 100 & 0.1 & 0.08 & 1.568 \\
5 & 400 & 0.1 & 0.2 & 1.983 \\
6 & 400 & 0.1 & 0.08 & 1.458 \\
7 & 100 & 0.15 & 0.14 & 2.296 \\
8 & 400 & 0.05 & 0.14 & 1.774 \\
9 & 100 & 0.05 & 0.14 & 1.975 \\
10 & 100 & 0.1 & 0.2 & 2.561 \\
11 & 250 & 0.1 & 0.14 & 1.701 \\
12 & 250 & 0.15 & 0.08 & 1.534 \\
13 & 250 & 0.1 & 0.14 & 1.644 \\
14 & 400 & 0.15 & 0.14 & 1.874 \\
15 & 250 & 0.1 & 0.14 & 1.671 \\
16 & 250 & 0.15 & 0.2 & 2.274 \\
17 & 250 & 0.1 & 0.14 & 1.681 \\
\hline
\end{tabular}

Table 7 Analysis of variance of different Sa models

\begin{tabular}{cccccccc}
\hline $\begin{array}{c}\text { Process } \\
\text { order }\end{array}$ & Model & Lack-of-fit & $\begin{array}{c}\text { R-Squar } \\
\text { ed }\end{array}$ & $\begin{array}{c}\text { R-Squared } \\
(\text { adj })\end{array}$ & $\begin{array}{c}\text { Pred } \\
\text { R-Squar } \\
\text { e }\end{array}$ & $\begin{array}{c}\text { Adeq } \\
\text { precision }\end{array}$ & $\begin{array}{c}\text { C.V. } \\
\%\end{array}$ \\
\hline Liner & Significant & Significant & 0.8126 & 0.7694 & 0.6731 & 13.898 & 8.59 \\
2FI & Significant & Significant & 0.8533 & 0.7653 & 0.5182 & 10.874 & 8.67 \\
$\begin{array}{c}\text { Quadrati } \\
\text { c }\end{array}$ & Significant & $\begin{array}{c}\text { Not } \\
\text { Significant }\end{array}$ & 0.9967 & 0.9923 & 0.9639 & 55.699 & 1.56 \\
\hline
\end{tabular}

According to the selection principle and applicability of the model, the Quadratic model is selected as the research object, and the influence of cutting parameters on the surface roughness formed by the 3D-USVT method is analyzed.

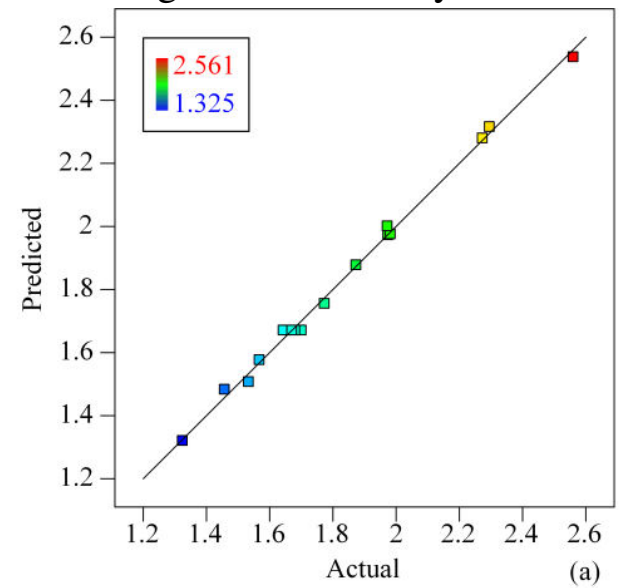

(a) Experimental results and predicted values

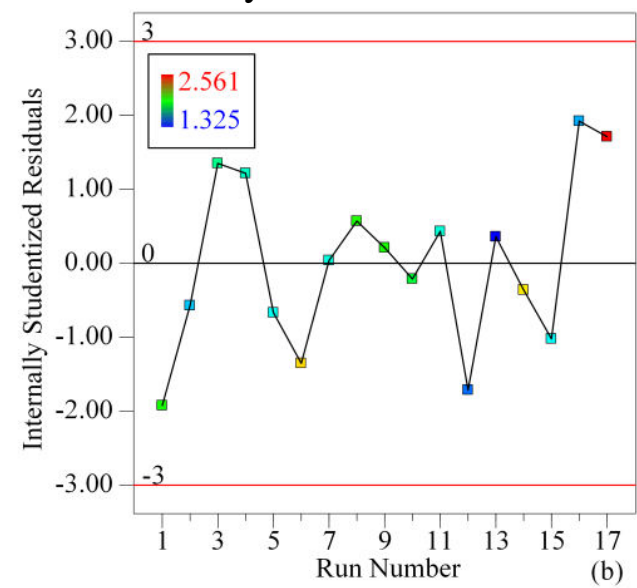

(b) Internal standard residual 


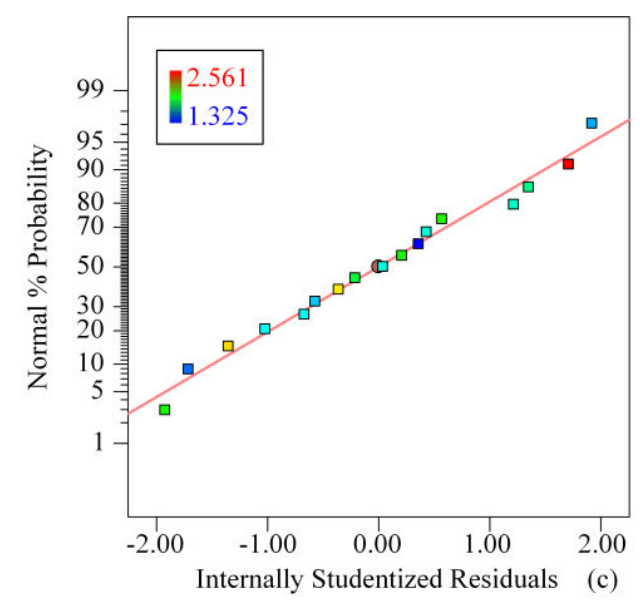

(c) Normal probability

Fig. 10 Applicability of Sa model
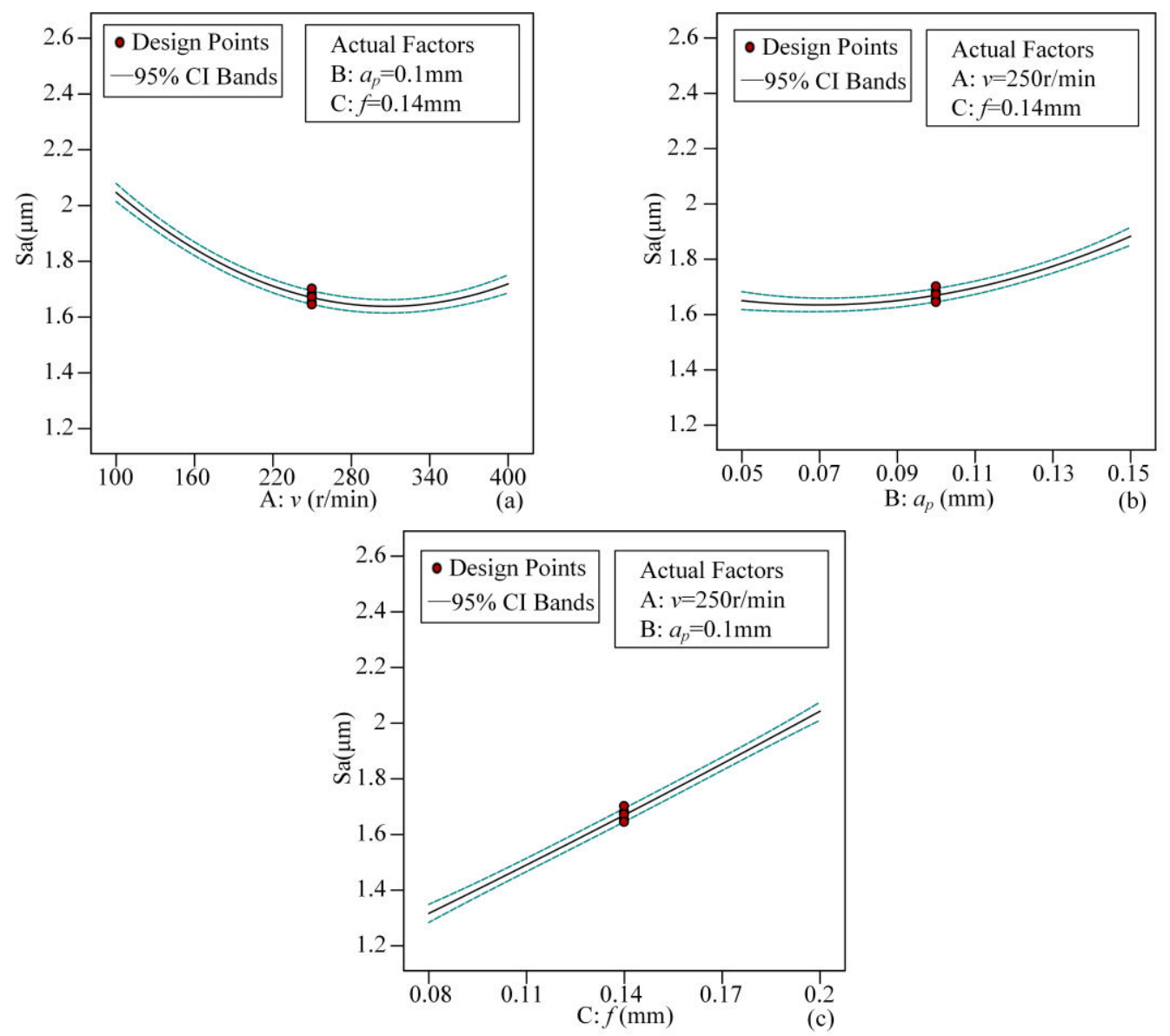

Fig. 11 Single-factor effect diagram of $\mathrm{Sa}$

Fig. 11 shows that the Sa obtained by the 3D-USVT cutting method decreases first and then increases with the increase of the $v$, and reaches the minimum value at $v=$ $320 \mathrm{r} / \mathrm{min}$ (Fig. 11(a)). With the increase of the $a_{p}$, Sa increases nonlinearly and slowly (Fig. 11(b)). Sa increases linearly with the increase of the feed, and the changing trend is faster than the $v$ and $a_{p}$ (Fig. 11(c)). The reasons for the above trends will be discussed in section 3.2 of this article, so I won't go into too much detail here. It can be seen that the effect of cutting parameters on the surface roughness of the 
workpiece obtained by the 3D-USVT cutting method is the same as that of the CT cutting method.
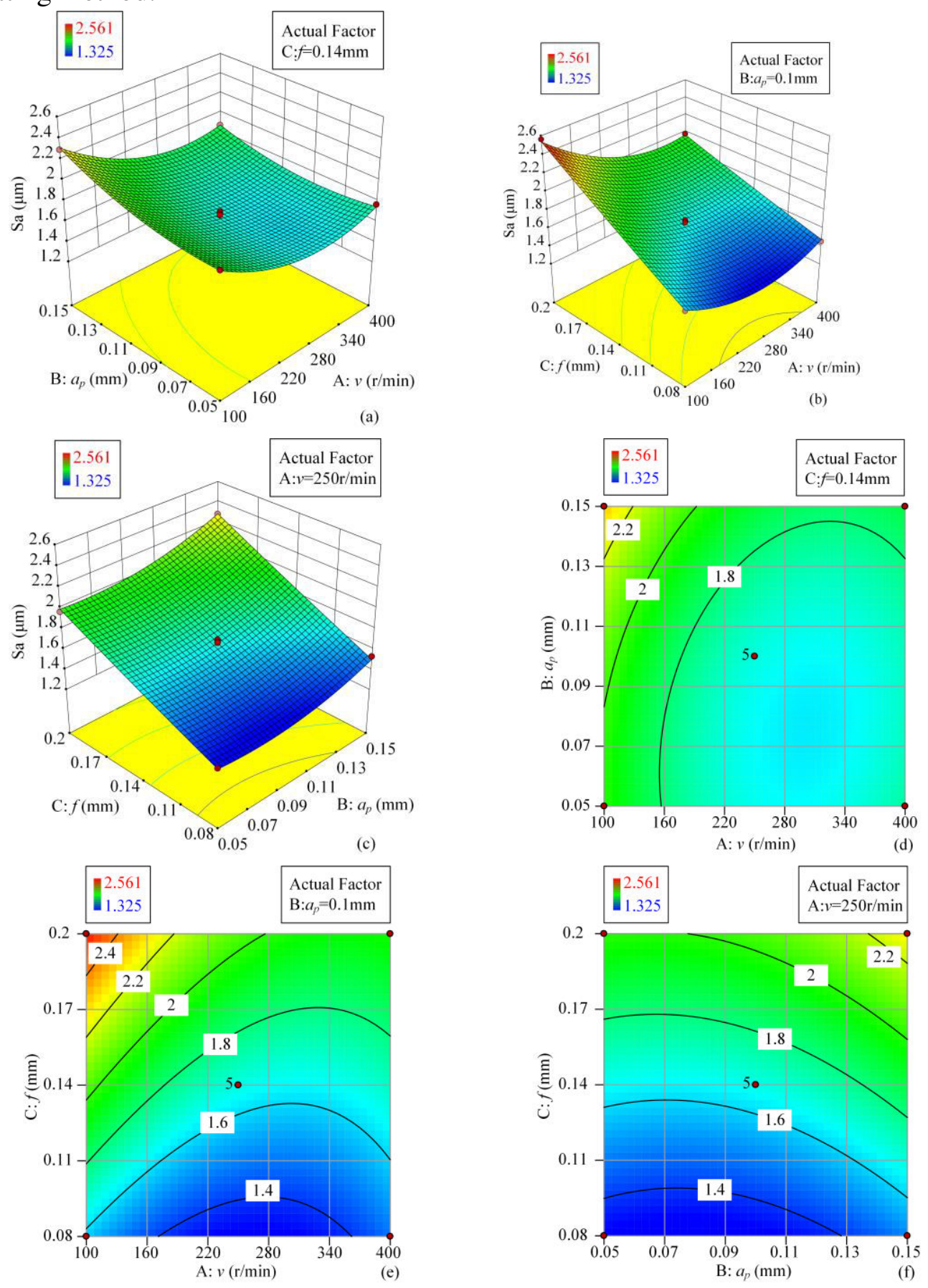

Fig. 12 Response surface plot and contour plot of Sa under the action of cutting parameters

Fig. 12(a) shows that as the $a_{p}$ increases, Sa increases. When the $v$ increases, as the $a_{p}$ increases, the rate of change of Sa gradually becomes slower. It can be thought that the greater the $v$, the smaller the effect of $a_{p}$ on Sa. As the $v$ increases, Sa first decreases and then increases. When the $a_{p}$ increases, as the $v$ increases, the rate of change of Sa slightly increases. It can be thought that the greater the $a_{p}$, the more obvious the effect of the $v$ on Sa.

Fig. 12(b) shows that as the $f$ increases, Sa becomes larger. When the $v$ increases, 
with the increase in the $f$, the rate of change of Sa gradually becomes slower. It can be observed that the greater the $v$, the smaller the influence of the $f$ on Sa. As the $v$ increases, $\mathrm{Sa}$ first decreases and then increases. When the $f$ increases, as the $v$ increases, the rate of change of Sa slightly increases. It can be seen that the greater the $f$, the more obvious influence of the $v$ on Sa.

Fig. 12(c) shows that as the $a_{p}$ increases, Sa becomes larger. When the $f$ increases, as the $a_{p}$ increases, the rate of change of Sa gradually becomes obvious. It can be seen that the larger the $f$, the more obvious influence of the $a_{p}$ on Sa. As the $f$ increases, Sa becomes larger. When the $a_{p}$ increases, with the increase of the $f$, the rate of change of Sa slightly increases. It can be seen that the greater the $a_{p}$, the more obvious influence of the $f$ on Sa.

It can be seen that there is obvious interaction among $v, a_{p}$, and $f$. Therefore, when selecting the cutting parameters of 3D-USVT, the effects of $v, a_{p}$, and $f$ on surface roughness should be comprehensively considered.

Fig. 12(d) shows that the influence of $v$ on Sa is more significant than that of $a_{p}$. From Figs. 12(e) and 12(f), it can be seen that the influence of $f$ on Sa is more significant than that of $v$ and $a_{p}$. It can be concluded that the order of influence of changing cutting speed $(v)$, depth of cut $\left(a_{p}\right)$, and feed $(f)$ on Sa is $f>v>a_{p}$.

In order to obtain the best response under the interaction of different factors, set the target value of Sa to the minimum, and set the cutting speed $(v)$, depth of cut $\left(a_{p}\right)$, and feed $(f)$ to the unconstrained state. The obtained cutting parameters obtained are shown in Table 8.

Table 8 optimal cutting parameters

\begin{tabular}{ccc}
\hline$v(\mathrm{r} / \mathrm{min})$ & $a_{p}(\mathrm{~mm})$ & $f(\mathrm{~mm})$ \\
\hline 277.79 & 0.069 & 0.084 \\
\hline
\end{tabular}

\section{Comparison and discussion of experimental results}

This section discusses the processing performance of the researched equipment through theoretical analysis and experimental results. First, the influence of the tool tip on the residual geometric height of the machined surface when cutting the workpiece under four different cutting methods of CT, 1D-UVT, UEVT, and 3D-USVT was discussed, and the residual geometric height of the four machining processes was compared. Then, under the same cutting conditions, by changing the cutting parameters $\left(v, a_{p}\right.$, and $f$ ), the variation trend of workpiece surface roughness under four different machining processes is discussed.

\section{1 theoretical analysis of surface roughness}

When cutting the workpiece, the surface roughness of the machined surface of the workpiece can be expressed as

$$
R_{t h}=R_{t e w}-s+w+\varepsilon_{t}
$$

where $R_{\text {tew }}$ represents the contribution of tool copying to the surface roughness, $s$ indicates the contribution of the elastic recovery of the workpiece material to the surface roughness, $w$ represents the contribution of the plastic side flow of the workpiece material to the surface roughness, and $\varepsilon_{t}$ is the contribution of surface 
defects in the workpiece material to the surface roughness.

The contribution of the tool to the surface roughness of the workpiece can be expressed as

$$
R_{\text {tew }}=r_{\varepsilon}-\sqrt{r_{\varepsilon}^{2}-\frac{f^{2}}{4}} \approx \frac{f^{2}}{8 r_{\varepsilon}}
$$

The height of the plastic side flow convex peak of the machined surface of the workpiece can be expressed as [47]

$$
w=1.32 \sqrt{0.46 \rho r_{\varepsilon} \cos ^{-1}\left(1-\frac{R_{\text {tew }}}{r_{\varepsilon}}\right)}
$$

where $\rho$ indicates the fillet radius of the cutting edge, and $r_{\varepsilon}$ represents the tool tip fillet radius.

The elastic rebound height of the processed surface material of the workpiece can be expressed as [48]

$$
s=c r_{\varepsilon}\left(1-\varepsilon_{p}\right)
$$

where $c$ represents dimensionless coefficient, and $\varepsilon_{p}$ is the plastic strain of the workpiece material.

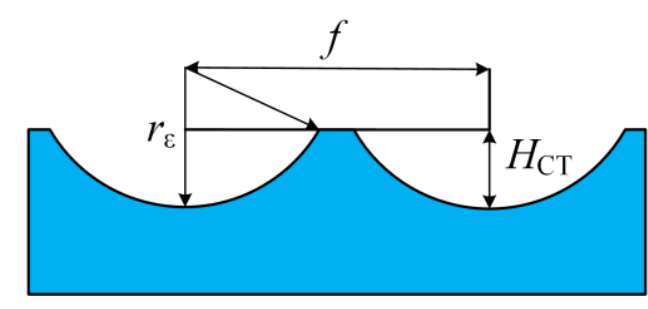

(a) $\mathrm{CT}$

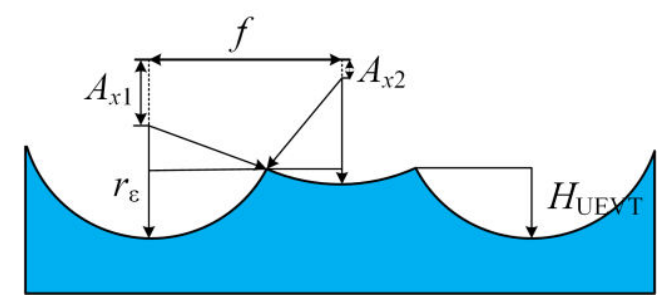

(c) UEVT

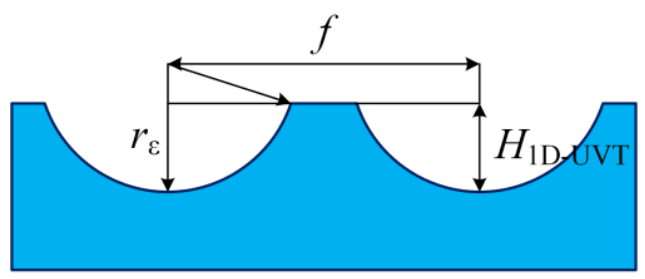

(b) 1D-UVT

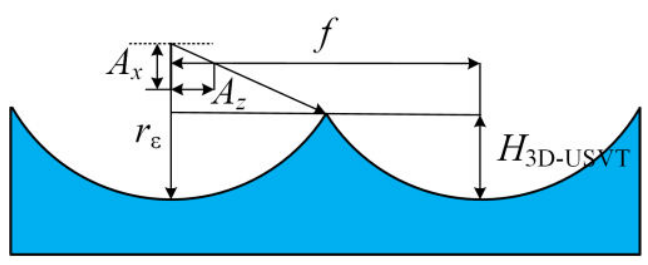

(d) 3D-USVT

Fig. 13 The geometric relationship diagram of two adjacent tool paths

From Fig. 13(a), it can be concluded that the contribution of tool copy to the residual geometric height of the workpiece when CT cutting the workpiece is

$$
2 \sqrt{r_{\varepsilon}^{2}-\left(r_{\varepsilon}-H_{C T}\right)^{2}}=f
$$

The workpiece was processed in CT, the tool is always in contact with the chips and the workpiece. The cutting heat generated during the cutting process causes the tool tip and the workpiece material to have a thermal expansion effect, and the tool tip fillet radius and the workpiece material are thermally expanded. According to formula (4), it can be seen that the part of the machined surface $R_{\text {tew }}$ formed by CT cutting decreases with the increase of the fillet radius, but the thermal expansion effect will not only produce gradient expansion of the tool tip, cause the expansion coefficient of the tool in the depth of cut direction to be greater than the feed direction but also 
increased tool wear. Under the same cutting conditions, the amount of workpiece material removed by $\mathrm{CT}$ in the depth of cut will increase, and the residual peak and valley height of the machined surface of the workpiece is significantly greater than the theoretical value. According to equations (5) and (6), it can be seen that the plastic side flow and elastic recovery in the turning process both increase significantly with the increase of the tool fillet radius. Due to its own cutting characteristics, the cutting heat and cutting force generated in the cutting process of 1D-UVT are significantly lower than those of CT. The plastic side flow, elastic recovery phenomenon, and lathe vibration phenomenon of the workpiece material will all be reduced, the separation characteristics of the tool and the chips increase the overlap of adjacent cutting paths, the overlapping area of adjacent cutting paths is cut repeatedly, and the residual height is reduced. However, the ironing effect of the tool flank on the machined surface reduces the elastic recovery of the machined surface material, and to a certain extent prevents the reduction of the residual geometric height of the workpiece surface. Overall, compared with CT, the surface roughness of the machined surface of 1D-UVT cutting is significantly smaller and closer to the theoretical value. Zong et al. [49] believe that material expansion will increase with the increase of polishing and friction, thereby increasing the surface roughness of the workpiece. The high-frequency repeated ironing effect when 1D-UVT is cutting the workpiece will not only increase the surface roughness, but the flank of the tool will also produce micro-cutting of the processed surface material during the ironing process, resulting in surface wear and surface fatigue on the processed surface, thus reducing the machining accuracy of the workpiece.

When cutting a workpiece with ultrasonic elliptical vibration-assisted turning (UEVT), due to the difference in phase difference in the vibration direction, or at the starting point of the adjacent cutting trajectory, the position of the vibration trajectory of the tool is different. This results in a difference in the surface geometry of the machined workpiece, for this purpose, the coefficient $M$ is introduced

$$
M=2 \omega \mathrm{S} / v=p+q
$$

where $S$ represents the total cutting stroke of the tool when the workpiece rotates once, $v$ indicates the rotation speed of the workpiece, $p$ is an integer, and $q$ is a decimal.

When $p \neq 0$ and $q=0$, the residual height of the machined surface formed by UEVT is the largest. At this time, the contribution of the tool copy to the machined surface of the workpiece can be expressed as

$$
R_{\text {tew }}=H_{U E V C}=A_{y}+H_{C T}
$$

where $A_{y}$ represents the vibration texture height of the tool in the $a_{p}$ direction, $H_{\mathrm{CT}}$ indicates the residual height of the tool in the feed direction, that is, the geometric height of the machined surface formed by CT cutting.

When $p \neq 0$ and $q=0.5$, the residual height of the machined surface formed by UEVT is small. At this time, the contribution of cutting tool scoring to the machined surface of the workpiece can be expressed as

$$
\sqrt{r_{\varepsilon}^{2}-\left(r_{\varepsilon}-H_{U E V C}-A_{x 1}-A_{x 2}\right)^{2}}+\sqrt{r_{\varepsilon}^{2}-\left(r_{\varepsilon}-H_{U E V C}-A_{x 2}\right)^{2}}=f
$$

where $A_{\mathrm{x} 1}$ represents the distance between the tool tip fillet center and the centerline of the track when the tool is at the lowest point of a single vibration trajectory, and $A_{\mathrm{x} 2}$ 
indicates the distance between the center of the tool tip fillet and the centerline of the track when the tool tip is cut to half of the net cutting time of a single cycle.

In the cutting process of UEVT, the tool is completely separated from the workpiece and the chips, and the cutting heat and cutting force generated are significantly reduced compared with CT and 1D-UVT. Due to the smaller cutting heat and cutting force, the thermal expansion effect of the tool and the workpiece is reduced, lathe vibration is also greatly reduced, which reduces the waviness of the machined surface. The instantaneous impact force of the tool also weakens the plastic side flow of the material and improves the machining accuracy of the workpiece. According to equation (9), it can be seen that when the instantaneous vibration form of the tool is the same at the starting point of the adjacent cutting path, there is no phase difference between adjacent cutting paths, at this time, the surface geometry height of the machined surface formed by the UEVT cutting workpiece is significantly greater than the CT. When the instantaneous vibration form of the tool is different at the starting point of each cutting path, there is a phase difference between adjacent cutting paths. According to equation (8), only when $n=0.5$, the surface geometry height of the machined surface be the lowest. According to equations (7) and (10), it can be seen that the residual geometric height of the machined surface formed by UEVT cutting at this time is significantly lower than that of CT and 1D-UVT.

When $p \neq 0, q \in[(0,0.5) \cup(0.5,1)]$, the contribution of UEVT tool scoring to the residual geometric height of the workpiece surface is between equation (9) and equation (10).

From Fig. 13(d), it can be concluded that the contribution of 3D-USVT tool scoring to the residual geometric height of the workpiece surface can be expressed as

$$
2 \sqrt{r_{\varepsilon}^{2}-\left(r_{\varepsilon}-H_{3 D-U V C}-A_{x}\right)^{2}}=f
$$

where $A_{x}$ represents the distance between the center of the tool tip fillet and the centerline of the path when the tool is at the lowest point of the vibration path.

Similar to UEVT, when 3D-USVT cuts the workpiece, the tool, and the workpiece are completely separated, the cutting heat and cutting force are reduced, and the thermal expansion of the tool and the workpiece and the vibration of the lathe has basically disappeared. Comparing with equation (10) and equation (11), it can be found that $H_{3 \mathrm{D}-\mathrm{USVT}}$ is slightly smaller than $H_{\mathrm{UEVT}}$, and it can be seen that the residual geometric height of the machined surface produced by 3D-USVT cutting is smaller than the minimum residual geometric height produced by UEVT cutting. It can be seen from Fig. 13(d) that when cutting the workpiece in 3D-USVT the tip of the tool vibrates not only in the direction of the depth of cut but also in the direction of the feed. This increases the width of the cutting trajectory in the feed direction, thereby increasing the coincidence of adjacent two cuts, the residual height generated by the tool on the adjacent cutting trajectory is partially cut, and the residual height is reduced. There is also vibration in the cutting speed direction of the tool, and the ultrasonic vibration in the three directions is coupled with each other. According to Fig. 5(d), it can be seen that the depth of the concave surface formed by the tool tip in the $a_{p}$ direction is significantly reduced, and in the same cycle, the number of grooves 
formed by the tool tip in the cutting track direction will also increase. Comparing with UEVT, this not only reduces the surface roughness of the machined surface, but also increases the lubricating oil contact area of the workpiece in later use, and extends the service life of the workpiece.

\subsection{Experiments show the changing trend of surface roughness}

This section uses a single factor analysis method to turn 304 stainless steel using common turning (CT) and ultrasonic vibration-assisted turning (1D-UVT, EUVC, 3D-USVT) to obtain different parameters $\left(v, a_{p}\right.$, and $\left.f\right)$ the surface roughness and its changing law. To better analyze the influence of different processing methods on surface roughness, 1D-UVT [12], UEVT [43], and 3D-USVT all adopt the best vibration parameters corresponding to each processing method.

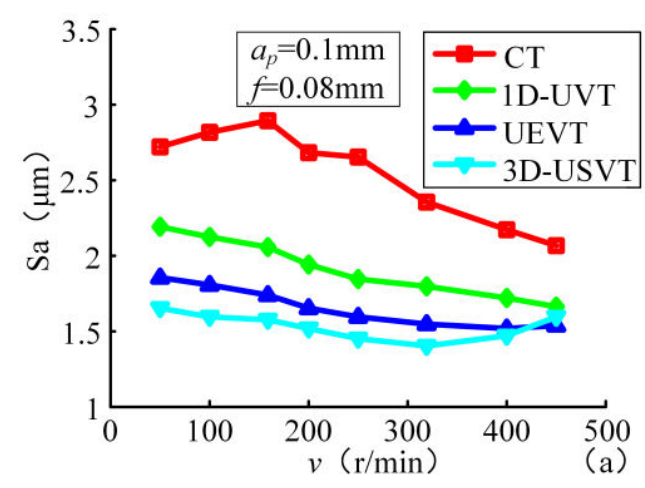

(a) The effect of $v$ on Sa

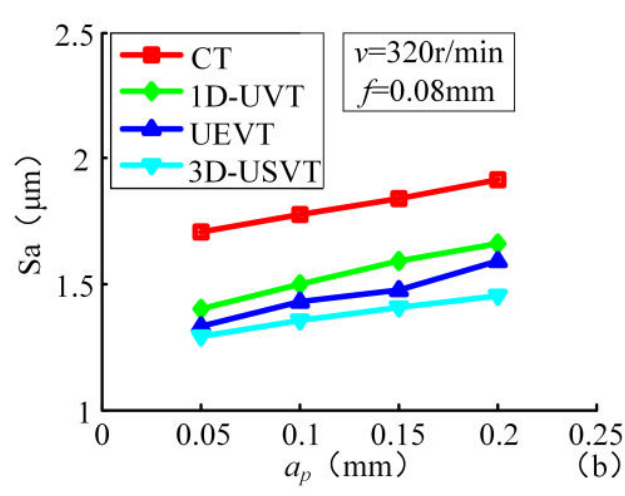

(b) The effect of $a_{p}$ on Sa

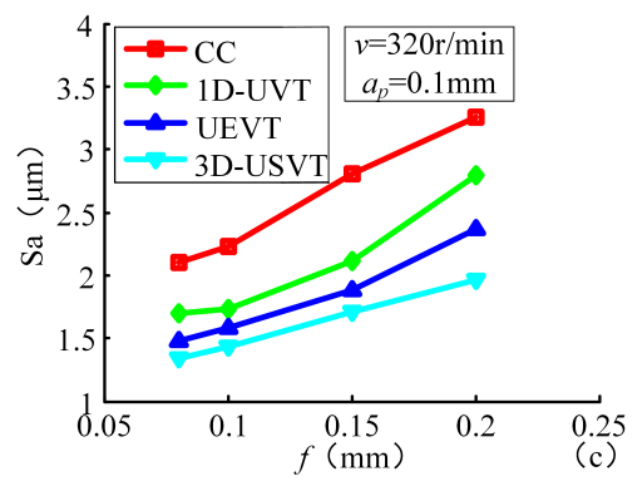

(c) The effect of $f$ on Sa

Fig. 14 The influence of cutting parameters on the surface roughness of the workpiece

Fig. 14(a) shows that with the increase of the $v$, the Sa values obtained by the four cutting methods all decrease non-linearly. This is because, with the increase of the $v$, the plastic deformation of the workpiece material and the reduction of the plastic side flow reduce the peak and valley height of the processed surface micro-morphology, and the surface roughness becomes smaller. Fig. 14(a) shows that with the increase of $v$, the surface roughness of the machined surface obtained by CT is much greater than that of the other three cutting methods. This is because the tool with high-frequency vibration has high acceleration, which makes the workpiece produce micro elastic 
deformation and ductility processing [44] can reduce the waviness of the machined surface grooves, and can also inhibit the plastic side flow of the workpiece surface [50] and reduce the surface roughness. When $v$ is between $50 \mathrm{r} / \mathrm{min}$ and $200 \mathrm{r} / \mathrm{min}$, the value of Sa shows an upward trend. This may be because the built-up edge produced by CT processing 304 stainless steel at low speed falls off and adheres to the processed surface, thereby increasing increase the surface roughness of the workpiece. The Sa value of the machined surface under the three cutting modes of 1D-UVT, UEVT, and 3D-USVT changes relatively smoothly. During 1D-UVT cutting, the flank face of the tool does not separate from the workpiece, and the high-frequency repeated fretting ironing effect reduces the surface roughness of the machined surface of the workpiece, it will also cause the machined surface to wear and even produce some scratches, which leads to the deterioration of the accuracy of the machined surface. Due to its cutting characteristics, UEVT not only reduces tool wear but also improves the integrity of the machined surface. Fig. 14(a) shows that when $v$ is greater than 400r/min, the Sa value rises slightly, which may be due to the weakening of the separation characteristics of UEVT as the $v$ increases. Compared with the other three cutting methods, when the $v$ of 3D-USVT is less than $320 \mathrm{r} / \mathrm{min}$, the Sa value of 3D-USVT is the smallest, which verifies the correctness of the conclusion in 3.1; when $v$ is greater than $320 \mathrm{r} / \mathrm{min}$, the Sa value of 3D-USVT began to rise sharply. This may be because the higher $v$ not only causes the instantaneous impact force of the tool to increase and aggravate the wear of the tool but also caused the tool to generate additional vibration, which reduced the stability of the cutting system.

By changing the $a_{p}$ to cut the workpiece, it can be seen from Fig. 14(b) that with the increase of the $a_{p}$, the Sa under the four different cutting methods shows an upward trend. This is because as the $a_{p}$ increases, the plastic side flow phenomenon of the material intensifies, and the residual geometric height of the workpiece surface increases [49].

$$
F=\frac{\tau A_{c}}{\sin \varphi \cos (\varphi+\beta-\alpha)}=\frac{\tau b_{D} h_{D}}{\sin \varphi \cos (\varphi+\beta-\alpha)}
$$

where $\tau$ represents the shear stress, $A_{c}$ represents the cutting area, $b_{D}$ represents the cutting width, and $h_{D}$ represents the cutting thickness.

According to equation (12), it can be concluded that when other cutting conditions remain unchanged and the $a_{p}$ is increased, the cutting area will increase accordingly, resulting in greater deformation resistance and friction, higher cutting force, which will eventually lead to vibrations in the entire turning system, the surface roughness becomes larger.

Fig. 14(c) shows that with the increase of the $f$, the Sa under the four different cutting methods shows an upward trend, but the Sa change rate is slightly different. According to equations (4) and (12), it can be seen that when the feed amount is small, the residual geometric height and cutting force generated when cutting the workpiece are small. The cutting vibration is not obvious, and the Sa is small. When the $f$ is increased, it will not only lead to a larger cutting force, the stability of the turning system will be reduced, but also a significant spiral groove will be generated on the surface of the workpiece, reducing the surface finish of the workpiece. Fig. 14(c) 
shows that when the feed per revolution of the tool exceeds $0.1 \mathrm{~mm}$, the surface roughness of 1D-UVT increases faster than the other three cutting methods which maybe since the overlapping area of two adjacent cutting trajectories decreases with the increase of feed, and the ironing effect of the tool is not significant.

By comparing Fig. 14, it can be found that the effect of changing the $a_{p}$ on Sa is significantly lower than the effect of $v$ and $f$ on Sa. The changing trend of this phenomenon is the same in traditional turning and ultrasonic-assisted turning. In general, for the four different cutting methods (CT, 1D-UVT, UEVT, and 3D-USVT), the maximum surface roughness appears in CT cutting and the smaller the surface roughness value with the increase of the applied vibration direction. Compared with 1D-UVT and UEVT, the surface roughness value obtained by 3D-USVT is the smallest, and the reduction degree can be up to $45.5 \%$ compared with CT.

\section{Conclusion}

Cutting 304 stainless steel by the 3D-USVT, the influence of vibration parameters and cutting parameters on the 3D-USVT was studied, and the best parameter combination was established by the surface corresponding method. The surface roughness of CT, 1D-UVT, UEVT, and 3D-USVT cutting methods was analyzed theoretically, and the theoretical analysis results were checked by experiments. The experiments show that 3D-USVT has excellent cutting performance. The main conclusions are as following:

1. In terms of vibration parameters, the amplitude in three directions has an impact on the surface roughness obtained by the 3D-USVT. The horizontal vibration along the OX direction has the greatest impact on $\mathrm{Sa}$, the vertical vibration along the OY direction has the least impact on Sa. In terms of cutting parameters, feed $(f)$ has the greatest impact on $\mathrm{Sa}$, and depth of cut $\left(a_{p}\right)$ has the least impact on Sa. Optimal vibration parameters and optimal machining parameters of the 3D-USVT are determined.

2. The causes of surface roughness are analyzed, and according to the influence of UEVT on surface topography, the coefficient $M$ is introduced. When there is fractional part in the $M$, the surface roughness obtained by the CT method is the largest, followed by 1D-UVT, and 3D-USVT is the smallest. When there is no fractional part in the $M$, the surface roughness obtained by the CT method is the largest, followed by UEVT, and 3D-USVT is the smallest.

3. The experimental results show that the surface roughness obtained by the four cutting methods will increase with the increase of feed $(f)$ and depth of cut $\left(a_{p}\right)$, and decrease with the increase in cutting speed $(v)$. Among them, surface roughness obtained by $3 \mathrm{D}$-USVT is the smallest, indicating that this method has good processing performance.

Funding This project is supported by the National Natural Science Foundation of China (Grant No. 51875097).

Authors Contributions Jingwei Duan: methodology, experiment, validation, editing, and writing-original draft; Ping Zou: resources and supervision; Shiyu Wei, Rui Fang, 
and Liting Fang: investigation. All authors read and approved the final manuscript. Availability of data and materials All data used in the manuscript are available as submitted.

Code availability Not applicable

\section{Declarations}

Ethical Approval Not applicable

Consent to Participate Not applicable

Consent to Publish Not applicable

Competing Interests The authors wish to declare that there are no known competing interests related to this publication.

\section{References}

1. Bedi S S, Sahoo S P, Vikas B (2020) Influence of cutting speed on dry machinability of AISI 304 stainless steel. Materials Today: Proceedings 38(8):2174-2180

2. Hong H, Riga A T, Gahoon J M (1993) Machinability of steels and titanium alloys under lubrication. Wear 162-164(2):34-39

3. Tekiner Z, Yesilyurt S (2004) Investigation of the cutting parameters depending on process sound during turning of AISI 304 austenitic stainless steel. Materials \& Design 25(6):507-513

4. Xavior M A (2012) Evaluating the machinability of AISI 304 stainless steel using alumina inserts. Journal of Achievements in Materials \& Manufacturing Engineering 55(2):841-847

5. Skelton R C (1969) Effect of ultrasonic vibration on the turning process. International Journal of Machine Tool Design \& Research 9(4):363-374

6. Celaya A, Lacalle L, Campa F J (2013) Application of ultrasonics as assistance in machining operations 2(1):159-172

7. Celaya A, Lacalle L, Campa F J (2010) Ultrasonic Assisted Turning of mild steels. International Journal of Materials and Product Technology 37(1-2):60-70

8. Babitsky V.I (2003) Ultrasonically assisted turning of aviation materials. Journal of Materials Processing Technology 132(1-3):157-167

9. Babitsky V.I, Mitrofanov A.V, Silberschmidt V.V (2004) Ultrasonically assisted turning of aviation materials: simulations and experimental study. Ultrasonics 42(1-9):81-86

10. Nath C, Rahman M (2008) Effect of machining parameters in ultrasonic vibration cutting. International Journal of Machine Tools \& Manufacture 48(9):965-974

11. Zou P, Xu Y, He Y (2015) Experimental Investigation of Ultrasonic Vibration Assisted Turning of 304 Austenitic Stainless Steel. Hindawi Publishing Corporation Shock and Vibration Volume 2015(6):1-19.

12. Xu Y, Gao F, Zou P (2020) Theoretical and experimental investigations of surface roughness, surface topography, and chip shape in ultrasonic vibration-assisted turning of Inconel 718. Journal of Mechanical Science and Technology 34(9):3791-3806

13. Bai W, Bisht A, Roy A (2019) Improvements of machinability of aerospatial-grade Inconel alloys with ultrasonically assisted hybrid machining. The International Journal of Advanced Manufacturing Technology 101:1143-1156

14. Shamoto E, Moriwaki T (1994) Study on Elliptical Vibration Cutting. CIRP Annals $43(1): 35-38$ 
15. Zhang. J, Cui T, Ge C (2016) Review of micro/nano machining by utilizing elliptical vibration cutting. International Journal of Machine Tools \& Manufacture 106:109-126

16. Moriwaki T, Shamoto E (1995) Ultrasonic Elliptical Vibration Cutting. CIRP Annals Manufacturing Technology 44(1):31-34

17. Ma C, Shamoto E, Moriwaki T (2005) Suppression of burrs in turning with ultrasonic elliptical vibration cutting. International Journal of Machine Tools and Manufacture 45(11):1295-1300

18. Khajehzadeh M, Boostanipour O, Amiri S (2020) The influence of Ultrasonic Elliptical Vibration Amplitudes on Cutting Tool Flank Wear. Proceedings of the Institution of Mechanical Engineers Part B Journal of Engineering Manufacture 234(12):1499-1512

19. Suzuki N, Masuda S, Haritani M (2004) Ultraprecision micromachining of brittle materials by applying ultrasonic elliptical vibration tutting. Micro-Nanomechatronics and Human Science 2004:133-138

20. Ma C, Shamoto E, Moriwaki T (2004) Study of machining accuracy in ultrasonic elliptical vibration cutting. International Journal of Machine Tools \& Manufacture 44(12-13):1305-1310

21. Zhang X, Kumar A S, Rahman M (2011) Experimental study on ultrasonic elliptical vibration cutting of hardened steel using PCD tools. Journal of Materials Processing Technology 211:1701-1709

22. He Y, Zou P, Zhu W (2017) Ultrasonic elliptical vibration cutting of hard materials: simulation and experimental study. International Journal of Advanced Manufacturing Technology 91(1-4):363-374

23. Usman M M, Zou P, Tian Y (2020) Experimental investigation on surface functional indices in Ultrasonic Elliptical Vibration Cutting of C45 carbon steel. The International Journal of Advanced Manufacturing Technology 109(1):187-200

24. Ahn J H, Lim H S, Son S M (1999) Improvement of Micromachining Accuracy by 2Dimensional Vibration Cutting. Procaspe 20:150-153

25. Kim G D, Loh B G (2007) Characteristics of chip formation in micro V-grooving using elliptical vibration cutting. Journal of Micromechanics \& Microengineering 17(8):1458-1466

26. Kim G D, Loh B G (2007) An ultrasonic elliptical vibration cutting device for micro V-groove machining: Kinematical analysis and micro V-groove machining characteristics. Journal of Materials Processing Tech 190(1-3):181-188

27. Kim G D, Loh B G (2008) Characteristics of elliptical vibration cutting in micro-V grooving with variations in the elliptical cutting locus and excitation frequency. Journal of Micromechanics \& Microengineering 18(2):025002

28. Moriwaki T, Shamoto E (1995) Ultrasonic Elliptical Vibration Cutting. CIRP Annals Manufacturing Technology 44(1):31-34

29. Kim G D, Loh B G (2010) Machining of micro-channels and pyramid patterns using elliptical vibration cutting. International Journal of Advanced Manufacturing Technology 49(9):961-968

30. Jieqiong L, Mingchun L (2013) Tool path generation for fabricating optical freeform surfaces by non-resonant three-dimensional elliptical vibration cutting. Proceedings of the Institution of Mechanical Engineers, Part C: Journal of Mechanical Engineering Science 228(7):1208-1222

31. Shamoto E, Suzuki N, Tsuchiya E (2005) Development of 3 DOF Ultrasonic Vibration Tool for Elliptical Vibration Cutting of Sculptured Surfaces. CIRP Annals - Manufacturing Technology 54(1):321-324

32. Yang Z, Zhu L, Gao Z (2020) Review of ultrasonic vibration-assisted machining in advanced 
materials. International Journal of Machine Tools and Manufacture 156:103594

33. Kurniawan R, Ali S, Ko T J (2018) Modal simulation analysis of novel 3D elliptical ultrasonic transducer. IOP Conference Series: Materials Science and Engineering 324(1): 012063

34. Kurniawan R, Ko T J (2019) Surface topography analysis in three-dimensional elliptical vibration texturing (3D-EVT). International Journal of Advanced Manufacturing Technology 102(5):1601-1621

35. Sajjady S A, Abadi H, Amini S (2016) Analytical and experimental Study of topography of surface texture in ultrasonic vibration assisted turning. Materials \& Design 93:311-323

36. Lin J, Lu M, Zhou X (2016) Development of a Non-Resonant 3D Elliptical Vibration Cutting Apparatus for Diamond Turning. Experimental Techniques 40(1):173-183

37. Lu M, Zhou J, Lin J (2017) Study on Ti-6Al-4V Alloy Machining Applying the Non-Resonant Three-Dimensional Elliptical Vibration Cutting. Micromachines 8(10):306-322

38. Zhu Z, To S, Zhang S (2016) High-Throughput Generation of Hierarchical Micro/Nanostructures by Spatial Vibration-Assisted Diamond Cutting. Advanced Materials Interfaces 3(4):1500477

39. Zhu Z, To S, Ehmann K (2017) Design, analysis, and realization of a novel piezoelectrically actuated rotary spatial vibration system for micro-/nanomachining. IEEE/ASME Transactions on Mechatronics 22(3):1227-1237

40. Lin J, Zhou J, Lu M (2020) Design of Robust Adaptive Fuzzy Controller for a Class of Single-Input Single-Output (SISO) Uncertain Nonlinear Systems. Mathematical Problems in Engineering 2020:1-11

41. Du Y, Lu M, Wang H (2021) Parameter tuning of robust adaptive fuzzy controller for 3D elliptical vibration-assisted cutting. Mechanical Sciences 12(1):433-442

42. Zhang C, Song Y (2019) Design and kinematic analysis of a novel decoupled 3D ultrasonic elliptical vibration assisted cutting mechanism. Ultrasonics 95:79-94

43. Zhao H , Li S , Zou P (2017) Process modeling study of the ultrasonic elliptical vibration cutting of Inconel 718. International Journal of Advanced Manufacturing Technology 92(5):2055-2068

44. Sharma V, Pandey P M (2018) Experimental investigations and statistical modeling of surface roughness during ultrasonic-assisted turning with self-lubricating cutting inserts. Proceedings of the Institution of Mechanical Engineers Part E: Journal of Process Mechanical Engineering 232(6):709-722

45. Krizbergs J, Kromanis A (2006) Methods for prediction of the surface roughness 3D parameters according to technological parameters. 5th International DAAAM Baltic Conference 20-22:145-149

46. Shuang Y, John M, Songlin D(2019) Experimental investigation on the performance and mechanism of graphene oxide nanofluids in turning Ti-6Al-4V. Journal of Manufacturing Processes 43:164-174

47. Chen G, Liu X, Yue C (2010) Study on Causes of Material Plastic Side Flow in Precision Hard Cutting Process. Advanced Materials Research 97:1875-1878

48. He C L, Zong W J, Sun T (2016) Origins for the size effect of surface roughness in diamond turning. International Journal of Machine Tools and Manufacture 106:22-42

49. Zong W J, Huang Y H, Zhang Y L (2014) Conservation law of surface roughness in single point diamond turning. International Journal of Machine Tools \& Manufacture 84:58-63 
50. Kim J D, Choi I H (1997) Micro surface phenomenon of ductile cutting in the ultrasonic vibration cutting of optical plastics. Journal of Materials Processing Technology 68(1): 89-98 